\title{
LA INNOVACIÓN EN LAS MIPYMES MANUFACTURERAS: ESTUDIO COMPARATIVO ENTRE EL ECUADOR Y LA ARGENTINA ${ }^{1}$ INNOVATION IN MANUFACTURING MSMES: A COMPARATIVE STUDY BETWEEN ECUADOR AND ARGENTINA
}

Silvana Astudillo

\section{Resumen}

La presente investigación está orientada a comparar las actividades de innovación de las micro, pequeñas y medianas empresas del sector manufacturero del Ecuador y la Argentina. En las últimas décadas las economías latinoamericanas han avanzado hacia la innovación dentro de un escenario mundial de cambios tecnológicos, de competitividad y mercado. En este sentido, el artículo analiza las determinantes de la innovación (inputs), la introducción de las actividades de innovación (outputs) y los efectos de la innovación (firm performance) que realizan las empresas manufactureras de ambos países. Se utilizó la información de las encuestas empresariales de los años 2006 y 2010 del Banco Mundial a través de un análisis estadístico bivariado y multivariado. Los resultados manifiestan que la I+D es una actividad determinante en ambos sectores con una introducción mayor de actividades de innovación en el producto y en el proceso en la Argentina, y

\footnotetext{
${ }^{1}$ Este artículo constituye un resumen de la tesis de doctorado en Ciencias de la Administración (UNS) de Silvana Astudillo, dirigida por la Dra. Anahí Briozzo. La misma fue defendida y aprobada el 07/02/2018 (Expte. 3383/2013).

${ }^{2}$ Universidad de Cuenca-Ecuador. E-mail: silvana.astudillo@ucuenca.edu.ec
} 
con objetivos similares perseguidos por ambos sectores empresariales como la reducción de costos, la obtención de certificaciones, entre otros efectos.

Palabras clave: innovación, manufactura, Ecuador, Argentina.

\begin{abstract}
This research work aims to compare the innovative activities of the manufacturing sector in Ecuador and Argentina. In recent decades, Latin American economies have advanced towards innovation within a global scenario of technological development, increased competitiveness and market change. Using data from the business surveys conducted by the World Bank in 2006 and 2010, the determinants of innovation (inputs), the introduction of innovative activities (outputs) and the effects of innovation (firm performance) in manufacturing companies were studied through bivariate and multivariate statistical analysis. Results indicate that in both countries, $R \& D$ has a decisive impact on the innovation activities of micro, small and medium-sized enterprises. Although the effect is greater in Argentina, entrepreneurs in Ecuador pursue similar objectives such as cost reduction and certification obtainment, among others.
\end{abstract}

Keywords: innovation, manufacture, Ecuador, Argentina.

JEL: $031 ; 032$ 


\section{Introducción}

En Latinoamérica, el impulso a las micro, pequeñas y medianas empresas (MIPYMES) es uno de las principales objetivos de las políticas públicas. En estas condiciones, muchos estudios sobre las pequeñas empresas se han realizado en los países de la región y se ha generado una considerable atención a los aspectos que las caracterizan, con un especial interés a la innovación como un factor clave para la mejora de su competitividad.

El objetivo de esta tesis fue realizar una comparación de las actividades de innovación de la MYPYMES manufactureras ecuatorianas y argentinas a partir de la sección de innovación y competitividad de la Enterprise Survey que realiza el Banco Mundial en los años 2006 y $2010^{3}$, bajo la hipótesis de que existen diferencias entre ambos grupos de empresas en las actividades de innovación. Los estudios de comparabilidad de la innovación entre los sectores industriales de los países latinoamericanos se convierten en un insumo importante para determinar el esfuerzo de los países respecto de la inversión en innovación y para evaluar las políticas públicas de los países en torno a este tema.

Por lo general, los países latinoamericanos diseñan sus políticas con base en políticas internacionales y con referentes de casos exitosos de países desarrollados. A primera vista, las actividades de innovación que realiza el Ecuador comparado con otros países de América del Sur tienen diferencias cuantitativas y cualitativas y evidencia una economía con menor capacidad para innovar, y su comparabilidad se torna interesante porque aporta una nueva evidencia del proceso innovador del Ecuador e inserta al país en un marco analítico de diseño de políticas con referencia a otros países latinoamericanos con un perfil más avanzado en ciencia, tecnología e innovación como la Argentina. Las economías de los dos países se visualizan con diferente desarrollo relativo y diferente desempeño en las actividades de innovación; sin embargo, ambos países tienen esfuerzos similares en investigación y desarrollo.

\footnotetext{
3 La investigación utiliza los datos de la encuesta empresarial del Banco Mundial del año 2006 y 2010. Hasta la fecha de la elevación de la tesis, el Banco Mundial no había presentado nuevos datos. El 15 de noviembre de 2017 el Banco Mundial presenta la encuesta empresarial del año 2017, después de la elevación de la tesis.
} 
A fines de conocer los elementos de la innovación en las MIPYMES en un estudio comparativo entre el Ecuador y la Argentina, y tomando como referencia el sector manufacturero y la innovación en el producto y en el proceso, se busca responder las siguientes preguntas de investigación:

- ¿Qué características diferenciales presentan las MIPYMES manufactureras en relación con la innovación en el Ecuador y la Argentina?

- ¿Cuáles son las determinantes de las actividades de innovación de las MIPYMES manufactureras en el Ecuador y la Argentina?

- ¿Cuáles son los efectos que genera en las MIPYMES manufactureras, la incorporación de las actividades de innovación desde la percepción de los empresarios en ambos países?

El artículo se estructura de la siguiente manera. En el siguiente apartado, se desarrolla un resumen del marco teórico y la revisión de la literatura empírica. A continuación, se describe la metodología. Luego se presentan los resultados obtenidos y por último se exponen las consideraciones finales.

\section{Marco teórico y revisión de la literatura}

\subsection{La innovación}

En la dimensión histórica, Schumpeter (1934) define la innovación como el desarrollo de un nuevo producto o proceso por parte de una empresa y su introducción exitosa en el mercado, el desarrollo de nuevas formas de organizar los negocios, nuevas fuentes de aprovisionamiento o la explotación de nuevos mercados. Freeman (1975) señala que la innovación en productos implica un nuevo bien o la mejora de un existente y la innovación en procesos, la introducción de nuevos equipos o medios de producción. Nelson y Winter (1982, p. 128) señalan que la innovación es "un cambio de rutina y la implementación de un diseño de un nuevo producto o de una nueva manera de producir un producto". Kline y Rosenberg (1986, p. 279) definen innovación como "un nuevo producto, un nuevo proceso de producción, la sustitución de materiales en un producto, la reorganización de la producción, las funciones 
internas, o la distribución que conduzcan a una mayor eficiencia, una mejora de los instrumentos o métodos de hacer la innovación".

Lundvall (1992) sostiene que la innovación es un proceso sistémico que se realiza en la empresa dentro de un proceso de interacciones entre todos los actores de un contexto nacional.

Edquist (2001) señala que las innovaciones pueden ser de producto o de proceso. La innovación de producto incluye tanto bienes como servicios, es decir, lo que se está produciendo. Las innovaciones de proceso se pueden clasificar en tecnológicas u organizativas, esto es, según la manera en que los bienes y servicios se producen.

Uno de los conceptos más completos para definir la innovación -que constituye una de las aportaciones más importantes sobre innovación y la que adopta este estudio - es la proveniente de la tercera edición del Manual de Oslo de la OCDE (2005) Organización para la Cooperación y el Desarrollo Económicos. El manual indica que innovar es "utilizar el conocimiento y generarlo si es necesario, para crear productos, servicios o procesos que son nuevos para la empresa, o mejorar los ya existentes, consiguiendo con ello tener éxito en el mercado".

A su vez, el Manual de Oslo distingue los conceptos de innovación del producto y del proceso (OCDE, 2005, p. 58):

- Una innovación de producto se corresponde con la introducción de un bien o de un servicio nuevo significativamente mejorado, ya sea en cuanto a sus características o en cuanto al uso. Esta definición introduce la mejora significativa de las características técnicas, los componentes y los materiales, la informática integrada, la facilidad de uso u otras características funcionales. Las innovaciones de producto pueden utilizar nuevos conocimientos o tecnologías o basarse en nuevas utilizaciones o combinaciones de conocimientos o tecnologías ya existentes.

- Una innovación de proceso es la introducción de un nuevo o significativamente mejorado proceso de producción o de distribución. Ello implica cambios significativos en las técnicas, los materiales y/o los programas informáticos. Una empresa innovadora en cuanto a producto/proceso es 
una empresa que ha introducido un nuevo producto o proceso, lo ha mejorado significativamente durante el período de estudio.

Lundvall (2009) manifiesta que las diferencias en cuanto al desempeño económico nacional motivan los estudios comparativos. La innovación es un fenómeno fundamental e inherente y las empresas deben emprender actividades que apunten a la innovación con el exclusivo fin de preservar su funcionamiento. Fagerberg (2013) expresa que la innovación no es un fenómeno nuevo; de hecho, es posible argumentar que es tan antigua como la humanidad misma. Parece haber algo inherentemente "humano" acerca de la tendencia a pensar en formas nuevas y mejores de hacer las cosas y probarlas en la práctica.

Dutrénit et al. (2010) menciona que la innovación aparece como una propiedad emergente del sistema, sobre la cual operan mecanismos de selección y retención. El primer mecanismo está asociado a las instituciones que determinan el "éxito" de la innovación y el segundo a los mecanismos que posibilitan su copia y difusión. En este contexto, el papel de las instituciones (empresas, universidades, centros de investigación, entre otros) y de las actuaciones públicas (gobierno) tienen un gran impacto en las actividades de innovación, a través de la regulación de los derechos de propiedad intelectual, la facilitación de los instrumentos de financiación pública, la financiación directa de las universidades, los incentivos fiscales para las actividades empresariales y el fomento proactivo entre los agentes, entre otros.

\subsection{Los determinantes de la innovación (inputs)}

El estudio sistemático de la innovación inicia con las obras de Joseph Schumpeter (1912), quien define la innovación como el desarrollo de un nuevo producto o proceso por parte de una empresa y su introducción en el mercado, la introducción de un nuevo método de producción, la apertura de un nuevo mercado, el acceso a nuevas fuentes de suministro, la implantación de una nueva estructura en un mercado. Según Schumpeter, el origen de las innova- 
ciones son los expertos que colaboran en equipos de I+D en busca de nuevas soluciones (Lundvall, 2009).

Desde que se ha reconocido la importancia de las actividades de innovación en el crecimiento económico, se han realizado numerosos esfuerzos para analizar cuáles son los factores que contribuyen al desarrollo de estas actividades y al éxito de los procesos de innovación en las empresas (Gómez y Calvo, 2011). De hecho, las investigaciones que buscan determinar los inputs de la innovación en las MIPYMES (micro, pequeñas, y medianas empresas) son muy relevantes para la formulación de políticas públicas por la importancia de su representatividad en los países en vías de desarrollo. Recientemente, una serie de estudios empíricos emergen para analizar la innovación en las empresas y sus determinantes, así como su impacto en otros indicadores de la empresa (Goedhuys y Veugelers, 2012). Bartels et al. (2012) manifiestan que los determinantes de los sistemas nacionales de innovación pueden apoyar las políticas de innovación y el crecimiento económico en los países en desarrollo.

La innovación en el sector manufacturero es un proceso muy complejo que es impulsado por numerosos factores. Los países desarrollados y algunos países en desarrollo han avanzado en la medición de estos esfuerzos a través de las encuestas de innovación. En este sentido, Barletta y Suárez (2015) manifiestan que los países de la región comparten similitudes que los diferencian de los países más desarrollados y que demandan indicadores específicos, útiles y relevantes para el diseño, implementación y monitoreo de la política tecnológica.

Las determinantes internas de la innovación que se evidencian son:

(a) Tamaño. El tamaño de la empresa muestra un efecto positivo con la innovación (Janz et al., 2004; Chudnovsky et al., 2006; Becheikh et al., 2006; Laforet, 2008; Gonçalves et al,. 2008; Milesi et al., 2011; Álvarez y García, 2012). Sin embargo, Adeyeye (2015) manifiesta que el rol que cumple el tamaño en las actividades de innovación no está del todo claro, ya que el análisis del tamaño de empresa en cuatro tipos de innovación: producto, proceso, marketing, organización, mostró que no era significativo. Por otra parte, De Jong y Vermeulen (2006) afirman que el tamaño de la empresa no está relacionado con la introducción de nuevos productos. 
(b) Mercado. La orientación al mercado ejerce un efecto positivo sobre la capacidad de innovación (Janz et al., 2004; Rhee et al., 2010). La estrategia y la orientación al mercado están asociadas con la innovación (Laforet, 2008).

(c) Calidad. La certificación de calidad tiene un efecto negativo en la innovación del producto y un efecto positivo en las innovaciones de proceso (Terziovski y Guerrero, 2014). Adoptar sistemas de calidad tiene una influencia favorable en las capacidades de innovación (François et al., 2002).

(c) Experiencia del gerente. La investigación previa sugiere que los estilos de liderazgo de los gerentes pueden afectar significativamente la capacidad innovadora de la organización (Jung et al., 2003; Avermaete et al., 2004; De Jong y Vermeulen, 2006). La presencia de un propietario/gerente con un grado académico no está asociado con una alta innovación, sin embargo, su experiencia laboral en un entorno científico es importante (Romijn y Albaladejo, 2002).

(d) Antigüedad de las empresas. Algunos estudios han observado una relación negativa entre la antigüedad de la empresa y la innovación, lo que implica que las empresas son cada vez más incapaces de generar nuevos o importantes innovaciones a medida que envejecen (Sørensen y Stuart, 2000). Sin embargo, Jung et al. (2003) menciona un efecto positivo entre la antigüedad y la innovación.

(e) Investigación y desarrollo. La introducción de innovaciones en productos y procesos depende en gran medida de la compra de I+D, de la concesión de licencias, la adquisición de know-how, patentes y marcas registradas; la contratación de servicios de consultoría, y los acuerdos de transferencia tecnológica (Romijn y Albaladejo, 2002; Gonçalves et al., 2008). Raymond y St-Pierre (2010) evidencian que la I+D no es sinónimo de innovación y que la I+D en la innovación en el producto no es sinónimo de I+D en la innovación en el proceso. El Manual del Oslo (OCDE,2005) menciona que la I+D desempeña un papel crucial en el proceso de innovación, aunque una gran parte de las actividades de innovación no se basan en ella, pero precisan tanto trabajadores altamente cualifica- 
dos como interacciones con otras empresas y con las instituciones públicas de investigación, así como una determinada estructura organizativa.

(f) Exportaciones. La innovación incrementa cuando el porcentaje de exportaciones incrementa (Landry et al., 2002).

(g) Resultados anteriores. La capacidad de aprendizaje tiene un impacto significativo en la innovación y en el rendimiento (Tsai, 2001).

(h) Capital extranjero. La presencia de capital extranjero en la firma resulta ser un factor determinante en todas las actividades innovadoras (Alvarez y García, 2012). La necesidad de un financiamiento externo incrementa el esfuerzo por innovar (Bartoloni, 2013).

(i) Recursos humanos calificados. La calidad del conjunto del capital humano de la empresa no influye de manera concluyente en la probabilidad de generar innovaciones (López-Mielgo et al., 2012). A su vez, otros autores evidencian que el perfil de formación de la fuerza laboral de la empresa puede contribuir a su capacidad de innovación (Porter, 1990; Romijn y Albaladejo, 2002).

Por su parte, las determinantes contextuales de la innovación que se evidencian son las siguientes:

(a) Región. El entorno regional influye en el alcance y la calidad de actividades de innovación. Esta influencia depende, sin embargo, en gran medida de las características de las empresas y sus empresarios (Sternberg y Arndt, 2001).

(b) Tecnología. Existe un efecto positivo sobre la innovación cuando se establece una nueva tecnología (Lee, 1995; Madeira et al., 2015; Ma y Liu, 2016). Sin embargo, Dini et al. (2014) mencionan que la dirección de la innovación no es rígida ni predeterminada por la tecnología. Específicamente, con referencia a internet, existe una relación positiva entre la banda ancha y la innovación (Grazzi y Jung, 2016).

(c) Competencia. Los competidores suministran información y animan a las empresas a innovar (François et al., 2002). 


\subsection{Los efectos de la innovación (firm performance)}

En referencia a los efectos de la innovación, una aportación importante la realiza Schumpeter (1934), quien menciona los efectos positivos que entraña la generación de nuevos productos y procesos.

Posteriormente al postulado de Schumpeter, la teórica evolucionista señala que el capitalismo está caracterizado por un proceso evolutivo asociado a la innovación. Rosegger (1996) plantea un modelo lineal de innovación, que presenta actividades dentro del proceso de innovación como la I+D, la investigación aplicada y el desarrollo tecnológico; dentro de los resultados, encontramos los descubrimientos, los invenciones o prototipos; finalmente, como resultado de la inversión en el aprendizaje, luego se pueden detectar efectos como productividad, desempeño económico o beneficios sociales.

Por otro lado, una innovación significativa puede dar a una empresa una ventaja importante sobre otras (Nelson y Winter, 1982; Kline y Rosenberg, 1986).

Malerba y Orsenigo (1996), en un estudio en siete países sobre los patrones de innovación schumpeterianos, explican que existen diferencias importantes como consecuencia de los efectos específicos de cada país en relación con los sistemas nacionales de innovación y las historias específicas de las empresas e industrias en cada país.

Posteriormente, la teoría endógena del crecimiento muestra la ventaja del enfoque schumpeteriano y su tratamiento explícito de la innovación como una actividad económica distinta; se introduce el paradigma de innovación endógena en el manejo de las políticas que influyen en el crecimiento (Grossman y Helpman, 1994; Aghion et al., 1998).

Con Lundvall (1992) emerge el enfoque de los sistemas nacionales de innovación que sostiene que la innovación es un proceso sistémico con interacciones entre todos los actores de una nación. Con respecto a las ventajas comparativas de la innovación en los productos y en los procesos, Metcalfe (1998) establece que los resultados son diferentes para cada empresa por su desempeño innovador, es decir, no existen dos empresas que innoven de la misma manera ni a la misma velocidad. De hecho, Fagerberg (2004) concluye que los 
esfuerzos para promover la competitividad mediante la innovación rara vez pueden entenderse al margen de lo que otros están logrando al mismo tiempo. Este argumento sugiere que el desempeño innovador es diferente en las firmas, industrias, regiones y entre países.

Lundvall (2009) considera que el motivo fundamental por el cual los gobiernos nacionales implementan políticas de innovación se basa en el supuesto de que la innovación es un elemento clave para el crecimiento económico nacional.

Los estudios empíricos manifiestan que la innovación genera un impacto positivo en las empresas (Crépon et al., 1998; Lööf et al., 2001; Klomp y Van Leeuwen, 2001; Sandven y Smith, 2000; Gálvez Albarracín y García Pérez de Lema, 2012; Gunday et al., 2011). Hassan et al. (2013) sugieren la necesidad de una investigación comparativa de los efectos de la innovación cruzando las diferencias culturales. En esta línea, Dutrénit y Katz (2005) en un estudio de la innovación en Latinoamérica, plantean tener más conocimiento sobre la relación de los enlaces entre los esfuerzos de la innovación y resultados de innovación.

Dutrénit et al. (2007) manifiestan que existe una relación cercana entre ciencia, tecnología e innovación y el desempeño económico, e incluso recaban evidencia sobre cómo estos factores han influido en el crecimiento de las economías industrializadas. Sin embargo, todavía no es claro de qué manera se debe usar la ciencia, la tecnología y la innovación para el crecimiento económico y social en los países en desarrollo. En gran medida esto parece depender de la habilidad de esos países para construir gradualmente trayectorias de aprendizaje e innovación.

Dutrénit et al. (2016) expresan que la innovación genera crecimiento económico si desencadena cambios estructurales, se identifican con nuevos sectores (o clases de productos ampliamente definidas), mercados, aglomeraciones, grandes empresas multinacionales, y otras formas que se han denominado organizaciones de alto nivel que pueden ser concebidas como estructuras multiagentes (por ejemplo, redes, sistemas regionales o sectoriales de innovación).

Asimismo, Antonelli (2017) concluye que la innovación no solo afecta al mercado de factores y de productos, sino también a la complejidad organizada del sistema y el funcionamiento de la conectividad del conocimiento en térmi- 
nos de acumulación de las existencias de conocimiento y a la consecuente generación de externalidades del conocimiento con efectos positivos y negativos. En un estudio en América Latina, Crespi et al. (2016) evidencian que los beneficios de la innovación dependen del tipo de innovación, con mayores efectos para el producto que para la innovación de procesos.

Suárez (2013) manifiesta que las decisiones de las empresas con respecto a cómo afrontar el proceso de selección constituyen la estrategia competitiva de la empresa y afectan su estructura general y sus capacidades. La autora presenta de manera esquemática la relación entre los inputs, outputs e impacto del proceso innovativo.

En esta línea, Dosi et al. (1988) manifiestan que la innovación en el producto y en el proceso en las firmas o en las industrias implica diferentes oportunidades como beneficios económicos. Este aporte concuerda con los resultados de Božić y Radas, 2006, Evangelista y Vezzani, 2010, Gómez y Calvo, 2011 y Reichert y Zawislak, 2014. Respecto de este análisis, existe un cuestionamiento de Sandven y Smith (2000), que mencionan una relación entre la rentabilidad y la innovación, pero no necesariamente en términos de ganancia. Por otro lado, con otro planteamiento, existen investigaciones en las industrias manufactureras, que señalan que la innovación en el producto tiene un enlace débil con las ventas de las empresas (Yeh-Yun y Yi-Ching Chen, 2007; Gunday et al., 2011). En referencia a las exportaciones, otros autores señalan que las pymes que tienen un historial de innovación son más propensas a exportar (Caldera, 2010; Martins et al., 2015; Love y Roper, 2015; De Fuentes et al., 2015). Por otra parte, las innovaciones permiten a las empresas reducir los costes marginales y obtener una ventaja sobre los competidores (Božić y Radas, 2006; Caldera, 2010, Bayraktar et al., 2017).

En relación con el impacto de la innovación en el mercado, la innovación puede ampliar el mercado e impulsar el crecimiento de la industria de manera positiva. Sobre la base de este argumento, Drucker (1986) plantea que una innovación produce un cambio en el mercado o en la sociedad, lo que sugiere que las empresas pueden penetrar en nuevos mercados o tener una mayor cuota de mercado. Esta afirmación la plantean también Lööf et al., 2001; Klomp y Van Leeuwen, 2001; Gómez y Calvo, 2011; Brown y Guzmán, 2014. 
Con respecto a la relación de la innovación con el empleo, Pianta (2003) señala que la innovación en el producto tiene un impacto positivo en el empleo y la innovación en el proceso posee un efecto negativo. Peters, 2008 concuerda con que el efecto de la innovación de procesos en el empleo es negativo. Otro impacto de la innovación se presenta en la mejora de la calidad de los bienes y servicios y la obtención de certificaciones de calidad (Prajogo y Sohal, 2003; Thai Hoang et al., 2006). Los autores indican una relación positiva y significativa en particular de la innovación de procesos. Otro argumento de los efectos de la innovación como resultado de la actividad innovadora - formulado por el Banco Mundial y analizado por Gómez y Calvo (2011) en la Encuesta sobre Innovación Tecnológica en las empresas del Instituto Nacional de Estadística de España en el año 2006 - es la utilización de menos materiales y energía por unidad producida. Con respecto a la productividad, Busom y Vélez-Ospina (2017) manifiestan que en las empresas del sector manufacturero de Colombia, las innovaciones en el producto, proceso, mercadotecnia y organización están correlacionadas positivamente con la productividad.

\section{Metodología}

\subsection{Base de datos}

Las variables de interés de la innovación del producto, la innovación del proceso y las variables internas, externas, efectos y control en ambos sectores manufactureros se analizaron tomando como base la Enterprise Survey del Banco Mundial (WBES) de los años 2006 y 2010.

\subsection{Caracterización de la muestra}

Para la clasificación de las MIPYMES se utilizó para ambos países la clasificación del Reglamento al Código de la Producción, Comercio e Inversiones (Registro OficialTM, 2010) del Ecuador, que clasifica las empresas según cantidad de empleados en microempresa (1-9 empleados), pequeñas (10 a 49 empleados), medianas (50 a 199 empleados). Esto coincide con la clasificación de la Argentina, que clasifica a las MIPYMES en el sector industrial de la siguiente 
manera: las microempresas poseen hasta 10 empleados, las pequeñas tienen entre 10 y 50 trabajadores y las medianas son aquellas cuya dotación de personal se encuentra entre 51 y 200. En el año 2010, en la Argentina (Buenos Aires, Mendoza, Córdoba, Rosario, Chaco) la WBES incluye a 102 microempresas, 346 pequeñas empresas y 209 medianas empresas del sector manufacturero. En el Ecuador (Pichincha, Guayas, Azuay) se incluyen 19 microempresas, 47 pequeñas empresas y 33 medianas empresas del sector manufacturero, datos que se utilizaron para el análisis de outputs de la innovación. Para los inputs y la performance de la innovación se utilizaron los datos del panel 20062010 del Banco Mundial, que refleja un total de 317 MIPYMES en la Argentina y 56 en el Ecuador. En los datos de la encuesta empresarial, surge que el sector industrial con mayor representación es el de alimentos y las pequeñas empresas son las más representativas en el sector.

\subsection{Herramientas estadísticas}

\subsubsection{Herramientas de análisis para la comparación de la innovación entre países}

Se aplicaron pruebas paramétricas para la comparación de ambos países: la prueba chi-cuadrado de Pearson para las variables cualitativas y la prueba test t de student para las variables cuantitativas. Se consideró como significancia estadística el valor de significancia inferior a 0,10. Los análisis estadísticos se realizaron con el uso del comando svy, que incorpora las ponderaciones del muestreo estratificado, de forma que los resultados sean representativos de la población. El estudio tomó la mediana como ponderación, como sugiere el Banco Mundial.

\subsubsection{Herramientas de análisis de los determinantes de la innovación}

Para responder a la pregunta sobre cuáles son los determinantes de la innovación en la Argentina y en el Ecuador, en el estudio se distinguen dos tipologías de la innovación: innovación en producto e innovación en proceso (OCDE, 2005). Así, la probabilidad de innovar en el producto y en el proceso cons- 
tituye la variable dependiente que toma el valor 1 cuando la empresa genera innovaciones y 0 en caso de contrario. La probabilidad que una empresa genere innovaciones se analizará con el modelo Probit:

$$
\operatorname{Pr}(\mathrm{y}=1)=\int_{-\infty}^{\beta / \mathrm{x}} \phi(\mathrm{t}) \mathrm{dt} \phi=\phi\left(\beta^{\prime} \phi \mathrm{x}\right)
$$

Donde $\phi$ representa la función de distribución normal estándar, x es el vector de las variables independientes, en este caso las variables internas y externas que determinan la innovación, y $\beta^{\prime}$ es el vector de parámetros a estimar. Además se recogen los efectos marginales para ambos países:

$$
\frac{\partial \mathrm{E}(\mathrm{y} / \mathrm{x})}{\partial \mathrm{x}}=\phi\left(\beta^{\prime} \mathrm{x}\right) \beta
$$

\subsubsection{Herramientas de análisis para los efectos de la innovación}

Se modeló a la innovación en el producto y en el proceso como una variable endógena que es considerada en un modelo de regresión con dos ecuaciones. El problema de endogeneidad aparece cuando una variable independiente está correlacionada con el error, que es la parte no observada de la regresión. En este caso, puede esperarse que la innovación sea una variable endógena a la perfomance de la empresa, ya que puede estar relacionada con factores no observables que influyen también en la perfomance. El problema de endogeneidad de los regresores se puede controlar con el uso de variables instrumentales ${ }^{4}$ y de regresiones en dos etapas.

La investigación utiliza como metodología un análisis multivariado. Para las variables cuantitativas de los efectos de la innovación se utiliza el siguiente modelo de regresión de dos ecuaciones (Cameron y Triverdi, 2009, p. 467):

$$
\begin{gathered}
\text { Ec. } 1 y_{\mathrm{i}}=y_{i} \beta_{1}+X_{1 i} \beta_{2}+u_{i} \\
\text { Ec. } 2 y_{i}=X_{1 i} \Pi_{1}+X_{2 i} \Pi_{2}+v_{i}
\end{gathered}
$$

\footnotetext{
${ }^{4}$ Las variables endógenas equivalen a las variables dependientes de un modelo de regresión de una sola ecuación, y las exógenas a las variables $X$, o regresoras en tal modelo, en tanto las variables $X$ no estén correlacionadas con el término de error de esa ecuación. La variable instrumental es una variable representante para la variable regresada rezagada pero con la propiedad que no está correlacionada con el error (Gujarati y Porter, 2009).
} 
Donde $y_{i}$ es la variable dependiente para la observación $i$-ésima (efecto de la innovación), $y_{i}$ representa al regresor endógeno (innovación en producto/proceso), $X_{1 i}$ representa los regresores exógenos incluidos, $X_{2 i}$ representa los regresores exógenos excluidos. $u_{i}$ y $v_{i}$ son los términos de error con media cero, y la correlación entre $u_{i}$ y los elementos de $v_{i}$, son presumibles diferentes a cero.

Para las variables cualitativas de los efectos de la innovación se controla la endogeneidad de la variable innovación en dos ecuaciones probit, bajo la forma de seemingly unrelated bivariate probit. La primera ecuación estima la variable efecto de la innovación y la segunda ecuación, la probabilidad de la innovación y sus determinantes. El modelo puede escribirse así según Baum (2006, p. 271):

$$
\begin{aligned}
& \text { Ec. } 1 y_{1}^{*}=X_{1} \beta_{1}+u_{1} \\
& \text { Ec. } 2 y_{2}^{*}=X_{2} \beta_{2}+u_{2}
\end{aligned}
$$

Donde

$$
\left(\begin{array}{l}
u_{1} \\
u_{2}
\end{array}\right) \sim N\left\{\left(\begin{array}{l}
0 \\
0
\end{array}\right),\left(\begin{array}{ll}
1 & \rho \\
\rho & 1
\end{array}\right)\right\}
$$

Las variables observables a las dos variables latentes $y_{1}^{*}, y_{2}^{*}$ son $y_{1}, y_{2}$. Estas variables $y_{1}, y_{2}$ toman el valor 1 si las correspondientes variables latentes $y_{1}^{*}$, $y_{2}^{*}$ toman valores positivos, y cero. Una formulación del modelo es el seemingly unrelated bivariate probit, donde dos ecuaciones probit son vistas como un sistema y deben estimarse en forma conjunta si $\rho \neq 0$. El no rechazo de la hipótesis nula conduce a la realización de una de un modelo probit simple.

En la primera etapa del modelo de dos ecuaciones, los determinantes de la innovación se identificaron en un estudio comparativo utilizando la Enterprise Survey del Banco Mundial del panel 2006-2010 (Astudillo y Briozzo, 2015) y se muestran en la ecuación 1.

Ec. 1 : Innovación Producto $2010=f\left(I+D_{2006}, r r h h_{2006}\right.$, calidad $\left._{2006}, v_{i}\right)$ Ec. 1: Innovación Proceso ${ }_{2010}=f\left(I+D_{2006}, r_{r h h} 2006\right.$, calidad $\left._{2006}, v_{i}\right)$ 
Posteriormente, la siguiente etapa de la ecuación consiste en estimar la siguiente ecuación, Ec. 2, empleando los resultados de la Ec. 1 para instrumentar la innovación:

Ec. 2: efecto ${ }_{2010}=$

(estimaciones innovación producto y proceso(Ec. 1 ), variables control, $\mu_{\mathrm{i}}$ )

En donde, los efectos de la innovación (performance empresarial) están en función de la innovación del producto y del proceso y necesitan de variables instrumentales que no estén correlacionadas con $\mu$ pero que estén correlacionadas con v. Una variable que satisface estas condiciones es una variable instrumental que no esté correlacionada con el efecto de la innovación pero sí con la variable innovación.

Para estimar la ecuación 2, se utilizaron las variables control como tamaño, antigüedad, financiamiento externo, género del gerente, competencia, indicadas en la segunda etapa de cada regresión. Para el análisis de la endogeneidad de la innovación en las variables cuantitativas, se utiliza los tests de DurbinWu-Hausman (Baum, 2006), que consideran a la variable innovación como exógena. En este trabajo, para el análisis de endogeneidad se considera el test de Durbin que usa una estimación de la varianza del error basada en el modelo suponiendo que las variables que se están probando son exógenas. La aceptación de la hipótesis nula de exogeneidad de la innovación, es decir un test Durbin no significativo, conduce a la realización de una regresión en una sola etapa: la estimación de $y_{\mathrm{i}}$ mediante mínimos cuadrados ordinarios. A su vez, la endogeneidad de la innovación para las variables cualitativas se estima con el test de la hipótesis nula de $\rho$ (rho) $=0$ (Baum, 2006, p. 271). La aceptación de la hipótesis nula de exogeneidad, es decir un rho no significativo, conduce a la realización de una regresión en una etapa: la estimación $y_{1}^{*}$ mediante un modelo probit simple. 


\section{Resultados}

\subsection{Análisis de los descriptores cualitativos y cuantitativos de la innovación}

En la tabla 1 se aprecia la comparación de actividades de innovación con características cualitativas entre las MIPYMES manufactureras del estudio de ambos países.

Los hallazgos permiten establecer las diferencias con significancia estadística:

- El porcentaje de MIPYMES argentinas que introdujeron un nuevo o significativamente mejorado producto en los tres últimos años es del 68,36 \%, y de las empresas ecuatorianas un $47,45 \%$. El test de independencia entre la Argentina y el Ecuador indica un valor significativo, por lo que es posible afirmar que se observan diferencias entre sectores manufactureros. Existen mayor proporción de MIPYMES manufactureras que innovan en el producto en la Argentina que en el Ecuador. En ambos países existe el predominio de las innovaciones de producto por sobre el proceso.

- En cuanto a la introducción de un proceso nuevo o significativamente mejorado en los últimos tres años, existen diferencias entre países. Se observa que las MIPYMES argentinas manufactureras innovan en el proceso en un 58,35 \% y las MIPYMES ecuatorianas en un 42,47 \%. Además la encuesta dimensiona cómo se percibe la introducción de un nuevo proceso o un proceso mejorado en la industria. Aquí existe una diferencia significativa entre países. Se observa que las MIPYMES ecuatorianas consideran que sus procesos son nuevos en la industria en un porcentaje considerable $(81,53 \%)$ en comparación con las MIPYMES argentinas (43,63 \%).

- Las MIPYMES argentinas manufactureras que realizaron gastos de investigación y desarrollo en el año fiscal representaron en promedio el $50,01 \%$, en tanto que las MIPYMES ecuatorianas que invirtieron en este sentido alcanzaron en promedio el 33,73 \% de las empresas. A su vez, la diferencia entre países es significativa respecto de la realización de actividades de I+D. Las MIPYMES argentinas tienen más propensión a invertir en $\mathrm{I}+\mathrm{D}$ que las ecuatorianas. 
- El porcentaje de MIPYMES argentinas manufactureras que forman parte del estudio que usaron servicios o programas que soporten la innovación en los últimos tres años es del 30,63 \% para el caso argentino; en cambio, para el caso ecuatoriano es del $46,87 \%$. Esto significa que existe una diferencia importante entre países.

Por su parte, el análisis desarrollado permite identificar semejanzas en las actividades de innovación cualitativas entre ambos sectores manufactureros:

- En lo que respecta a la introducción de un bien o servicio nuevo o significativamente mejorado que son nuevos en el mercado, no existen diferencias significativas entre la Argentina y el Ecuador. El porcentaje para las empresas manufactureras argentinas del $47,94 \%$ y para las empresas ecuatorianas del 50,74\%.

- Por el lado de uso de instrumentos de propiedad intelectual, como el registro de patentes, se constata que no existe diferencia entre países. Las MIPYMES argentinas manufactureras realizaron el registro de patentes en un 5,98 \% y las MIPYMES ecuatorianas en un 6,45\%.

- El soporte del sector público a las actividades de innovación en lo que respecta al apoyo financiero o asistencia en los tres últimos años en las MIPYMES argentinas y las MIPYMES ecuatorianas no presenta una diferencia significativa entre ambos países, las medias en porcentajes son del $15,11 \%$ y el $8,90 \%$.

- En lo concerniente al porcentaje de participación en actividades de innovación con otras empresas de ciencia y tecnología en los últimos tres años es muy similar en ambos sectores manufactureros, con un promedio del $17,26 \%$ y el $16,07 \%$, respectivamente.

- El porcentaje de MIPYMES que realizaron gastos en la adquisición de licencias para la propiedad intelectual como patentes, diseños industriales, derechos de autor, marcas comerciales y servicios de consultoría no registra diferencias entre los sectores de ambos países. Para el caso ecuatoriano el promedio es del $32,76 \%$ mientras para el caso argentino del 20,56 \%. 
Tabla 1. Argentina y Ecuador: descriptivos cualitativos de las MIPYMES en torno a la innovación 2010

\begin{tabular}{|c|c|c|c|c|c|c|c|}
\hline & \multicolumn{3}{|c|}{ Argentina } & \multicolumn{3}{|c|}{ Ecuador } & \multirow[b]{2}{*}{ P-valor } \\
\hline Variables cualitativas & $\begin{array}{l}\text { No. } \\
\text { Obs. }\end{array}$ & Media & $\begin{array}{l}\text { Des. } \\
\text { Est. }\end{array}$ & $\begin{array}{l}\text { No. } \\
\text { Obs. }\end{array}$ & Media & $\begin{array}{l}\text { Des. } \\
\text { Est. }\end{array}$ & \\
\hline $\begin{array}{l}\text { MIPYMES que introdujeron un nuevo o } \\
\text { significativamente mejorado producto } \\
\text { en los tres últimos años (en porcentaje) }\end{array}$ & 647 & 68,36 & 45,54 & 94 & 47,45 & 50,20 & $0,0211^{* *}$ \\
\hline $\begin{array}{l}\text { MIPYMES que realizaron la introduc- } \\
\text { ción de un bien o servicio nuevo o } \\
\text { significativamente mejorado, que son } \\
\text { nuevos en el mercado en los tres últi- } \\
\text { mos años }\end{array}$ & 452 & 47,94 & 50,01 & 54 & 50,74 & 50,46 & 0,7941 \\
\hline $\begin{array}{l}\text { MIPYMES que realizaron un nuevo o } \\
\text { significativamente mejorado proceso, } \\
\text { en los tres últimos años (en porcentaje) }\end{array}$ & 645 & 58,35 & 49,33 & 94 & 42,47 & 49,69 & $0,0284^{* *}$ \\
\hline $\begin{array}{l}\text { MIPYMES que realizaron la introduc- } \\
\text { ción de un nuevo o significativamente } \\
\text { proceso para producir o distribuir } \\
\text { bienes o servicios, que son nuevos en la } \\
\text { industria (en porcentaje) }\end{array}$ & 354 & 43,63 & 49,66 & 43 & 81,53 & 39,26 & $0,0009^{* *}$ \\
\hline $\begin{array}{l}\text { MIPYMES que realizaron actividades } \\
\text { de investigación y desarrollo en el año } \\
\text { fiscal (en porcentaje) }\end{array}$ & 648 & 50,01 & 50,03 & 94 & 33,73 & 47,53 & $0,0446^{* *}$ \\
\hline $\begin{array}{l}\text { MIPYMES que han realizado registro } \\
\text { de patentes (en porcentaje) }\end{array}$ & 643 & 5,98 & 23,74 & 93 & 6,45 & 24,69 & 0,7070 \\
\hline $\begin{array}{l}\text { MIPYMES que realizaron gastos en la } \\
\text { adquisición de licencias para propiedad } \\
\text { intelectual como patentes, diseños } \\
\text { industriales, registros, } \\
\text { servicios de consultoría en el año fiscal } \\
\text { (en porcentaje) }\end{array}$ & 646 & 20,56 & 40,45 & 94 & 32,76 & 47,18 & 0,1276 \\
\hline $\begin{array}{l}\text { MIPYMES que han sido apoyadas por } \\
\text { el sector público (financiero o asisten- } \\
\text { cia) en actividades de innovación en los } \\
\text { tres últimos años (en porcentaje) }\end{array}$ & 651 & 15,11 & 35,84 & 94 & 8,90 & 28,62 & 0,1378 \\
\hline $\begin{array}{l}\text { MIPYMES que cooperaron en activida- } \\
\text { des de innovación con otras empresas } \\
\text { de ciencia y tecnología en los últimos } \\
\text { tres años (en porcentaje) }\end{array}$ & 638 & 17,26 & 37,82 & 94 & 16,07 & 36,92 & 0,8338 \\
\hline $\begin{array}{l}\text { MIPYMES que usaron servicios o pro- } \\
\text { gramas que soporten a la innovación en } \\
\text { los últimos tres años (en porcentaje) }\end{array}$ & 652 & 30,63 & 46,13 & 98 & 46,87 & 50,15 & $0,0425^{* *}$ \\
\hline
\end{tabular}

Fuente: elaboración propia con base en la Enterprise Survey del Banco Mundial (2010).Se indica en la media el porcentaje de empresas de cada país que posee la característica. Valores p calculados con el test de Pearson. 
La tabla 2 presenta el análisis de las características cuantitativas en torno a las actividades de innovación de las empresas manufactureras argentinas y ecuatorianas que formaron parte del estudio. Es importante señalar que para el análisis estadístico de estas variables se utilizó lo manifestado por Cameron y Triverdi (2009), que expone que las ponderaciones del muestreo estratificado deben tenerse en cuenta al calcular medias muestrales, a fines que sean representativas de la población. Los hallazgos permiten establecer las siguientes diferencias con significancia estadística:

- Con respecto a la antigüedad promedio que tienen las empresas, existe diferencia entre ambos países, las MIPYMES argentinas presentan una media de 29,74 años y las MIPYMES ecuatorianas de 20,29 años. Las empresas manufactureras argentinas son más antiguas en comparación con las empresas ecuatorianas.

- A su vez, los años de educación de los trabajadores muestran que existen diferencias entre ambos países, el valor de significancia estadística positiva indica que los trabajadores de las MIPYMES manufactureras ecuatorianas tienen más años de educación que las argentinas. La media para el caso ecuatoriano es de $11,36 \%$ y para el caso argentino de $10,25 \%$.

- El porcentaje de las compras en maquinaria, equipo, vehículos destinadas para la innovación del producto y para la innovación en el proceso en el año fiscal evidencia diferencia entre países, con un porcentaje mayor en el Ecuador (60,05 \%) en comparación con la Argentina (43,06 \%).

Por su parte, se manifiestan las siguientes semejanzas entre las MIPYMES argentinas y ecuatorianas:

- El porcentaje de gasto de I+D sobre ventas no presenta diferencias entre las MIPYMES argentinas y las ecuatorianas que forman parte del estudio. La media del porcentaje de I+D en las MIPYMES argentinas de la muestra es del 2,98\% y la media de las ecuatorianas es del 2,45\%.

- El análisis del porcentaje de las ventas destinado a la innovación en el producto en los últimos tres años muestra que no existen diferencias sig- 
nificativas entre países, con un porcentaje promedio de ventas destinado a la innovación de 24,65 \% en la Argentina y de 26,66 \% en el Ecuador.

- El porcentaje de gasto sobre ventas que las MIPYMES destinaron a la adquisición de patentes, diseños industriales, derechos de autor, marcas comerciales y servicios de consultoría en el año fiscal es del 4,30 \% para la Argentina y del 0,46 \% para el Ecuador, sin mostrar diferencias entre países. Los resultados de propiedad intelectual presentan porcentajes bajos en ambos países.

- En referencia a las exportaciones directas que realizan las empresas manufactureras de la muestra, no existen diferencias entre países. Las MIPYMES argentinas presentan un porcentaje en la media de sus exportaciones del 8,52 \% y las MIPYMES ecuatorianas del 5,30\%.

- El resultado del porcentaje del financiamiento realizado por las MIPYMES manufactureras a bancos, instituciones no financieras, créditos a proveedores señala que no existen diferencias entre países. Las MIPYMES argentinas presentan una media del $41,50 \%$ de financiamiento externo y las MIPYMES ecuatorianas del 51,02 \%

- En lo que concierne al número de trabajadores en la microempresa, pequeña y mediana empresas, no existen diferencias entre países. Los datos del estudio muestran que la media del número de empleados en la microempresa argentina es 7 trabajadores al igual que la microempresa ecuatoriana. La media de trabajadores en la pequeña empresa es de 23 para el caso argentino al igual que el ecuatoriano. Para la mediana empresa argentina la media de trabajadores es de 95 trabajadores y para la mediana empresa ecuatoriana es de 91 trabajadores.

Es importante destacar que ambos sectores manufactureros no tienen el mismo número muestral de empresas por aspectos de extensión territorial; sin embargo la estructura del promedio del número de empleados es similar. 
La innovación en las MIPYMES manufactureras: estudio comparativo...

Tabla 2. Argentina y Ecuador: descriptivos cuantitativos de las MIPYMES en torno a la innovación 2010

\begin{tabular}{|c|c|c|c|c|c|c|c|}
\hline & & Argentin & & & Ecuador & & \\
\hline Variables cuantitativas & $\begin{array}{l}\text { No. } \\
\text { Obs. }\end{array}$ & Media & $\begin{array}{l}\text { Des. } \\
\text { Est. }\end{array}$ & $\begin{array}{l}\text { No. } \\
\text { Obs. }\end{array}$ & Media & $\begin{array}{l}\text { Des. } \\
\text { Est. }\end{array}$ & P-valor \\
\hline Antigüedad en años & 650 & 29,74 & 20,23 & 98 & 20,29 & 13,96 & $0,0000^{*}$ \\
\hline $\begin{array}{l}\text { Porcentaje de gasto en I+D sobre las } \\
\text { ventas en el año fiscal dentro de las } \\
\text { MIPYMES }\end{array}$ & 278 & 2,98 & 5,47 & 34 & 2,45 & 2,84 & 0,5495 \\
\hline $\begin{array}{l}\text { Porcentaje del gasto sobre ventas de las } \\
\text { MIPYMES en la adquisición de patentes, } \\
\text { diseños industriales, derechos de autor, } \\
\text { marcas comerciales y servicios de consul- } \\
\text { toría en el año fiscal (en porcentaje) }\end{array}$ & 124 & 4,30 & 28,27 & 23 & 0,46 & 0,42 & 0,2756 \\
\hline $\begin{array}{l}\text { Porcentaje de las ventas destinadas a la } \\
\text { innovación en producto en los últimos } \\
\text { tres años }\end{array}$ & 425 & 24,65 & 22,48 & 53 & 26,66 & 22,25 & 0,5907 \\
\hline $\begin{array}{l}\text { Porcentaje de las compras en maquinaria, } \\
\text { equipo, vehículos destinada para la inno- } \\
\text { vación del producto y proceso en el año } \\
\text { fiscal (en porcentaje) }\end{array}$ & 381 & 43,06 & 42,78 & 53 & 60,05 & 44,62 & $0,0648^{* *}$ \\
\hline $\begin{array}{l}\text { Exportaciones Directas (porcentaje sobre } \\
\text { las ventas) }\end{array}$ & 649 & 8,52 & 19,80 & 98 & 5,30 & 18,14 & 0,2153 \\
\hline $\begin{array}{l}\text { Financiamiento (porcentaje del capital } \\
\text { financiado por Bancos, instituciones no } \\
\text { financieras, créditos de proveedores, } \\
\text { otros) }\end{array}$ & 646 & 41,50 & 38,35 & 96 & 51,02 & 41,44 & 0,1634 \\
\hline $\begin{array}{l}\text { Años de educación promedio de los } \\
\text { trabajadores }\end{array}$ & 608 & 10,25 & 2,30 & 93 & 11,36 & 2,26 & $0,0053^{*}$ \\
\hline Número total de trabajadores MIPYMES & 657 & 35,33 & 36,60 & 99 & 34,32 & 34,41 & 0,8384 \\
\hline $\begin{array}{l}\text { Número de trabajadores en la microem- } \\
\text { presa }\end{array}$ & 102 & 6,69 & 1,93 & 19 & 7,07 & 1,85 & 0,5560 \\
\hline $\begin{array}{l}\text { Número de trabajadores en la pequeña } \\
\text { empresa }\end{array}$ & 346 & 22,97 & 8,64 & 47 & 23,54 & 10,54 & 0,8355 \\
\hline $\begin{array}{l}\text { Número de trabajadores en la mediana } \\
\text { empresa }\end{array}$ & 209 & 94,50 & 44,57 & 33 & 91,22 & 35,27 & 0,6832 \\
\hline
\end{tabular}

Fuente: elaboración propia con base en la Enterprise Survey del Banco Mundial (2010). Se indica en la media el porcentaje de empresas de cada país que posee la característica. Valores p calculados con el test $t$ de Student.

Nota: $* * 5 \%$ de significancia, ${ }^{*} 10 \%$ de significancia 


\subsection{Análisis de los determinantes de la innovación (inputs)}

La tabla 3 presenta los resultados del modelo probit y el asterisco indica su probabilidad ( $\mathrm{P}>|\mathrm{z}|)$. Adicionalmente, se analiza los efectos marginales del modelo para cada país. El modelo se ajustó eliminando la variable ámbito de operación, que evidenció colinealidad. El estadístico $X^{2}$ indica que el modelo es significativo a un nivel de confianza del $95 \%$. Se determina el análisis probit poniendo como énfasis al país como regresor, en este caso Argentina toma el valor de 1 y Ecuador el valor de 0 . El estudio de los determinantes de la innovación en las MIPYMES manufactureras de la Argentina y del Ecuador evidencia variables internas que determinan la propensión a la innovación en el producto y en el proceso, la variable con un efecto positivo y significativo en ambos países y en las dos tipologías de la innovación es la investigación y el desarrollo. En la innovación en el producto no se revelan otras determinantes y en la innovación en el proceso aparecen asociadas variables internas de las empresas como los recursos humanos calificados a través de los programas de entrenamiento que realizan las empresas y la certificación de normas internacionales de calidad.

En referencia al país como regresor, la Argentina incrementa en 17,30 \% la probabilidad de innovar en los procesos con una significancia estadística positiva, lo que sugiere que la empresa manufacturera argentina tiene más probabilidad de innovar en los procesos que una empresa manufacturera ecuatoriana.

La variable región, que corresponde a las capital de la Argentina y el Ecuador, donde se ubican las MIPYMES manufactureras (Buenos Aires y Pichincha), ejerce un efecto negativo y significativo hacia la elección de la propensión de innovar. Por otro lado, las variables como el tamaño, la antigüedad, la experiencia del gerente, el género del propietario, el capital extranjero, los resultados anteriores de la empresa, las exportaciones, la competencia, la tecnología no son estadísticamente significativos en la propensión de la innovación del producto y del proceso.

Los efectos marginales en las variables internas y externas de la innovación evidencian que en el caso de la I+D, esta incrementa en 22,61 \% la probabilidad de innovar en el producto; por otro lado, la I+D incrementa en un 16,10\% la probabilidad de innovar en el proceso, siendo mayor la propensión de I+D 
en la innovación en el producto. Los recursos humanos incrementan en un $15,41 \%$ la probabilidad de innovar en los procesos y la adopción de sistemas de calidad incrementa en 18,27 \% la propensión a innovar en el proceso. En el caso de la región, la empresa que esté ubicada en la capital reduce la propensión a innovar en un 17,10 \% (tabla 17).

Tabla 3. Argentina y Ecuador: efectos marginales de las variables internas y externas de la innovación (2006-2010)

\begin{tabular}{|c|c|c|c|c|}
\hline \multirow[b]{3}{*}{ Variables } & \multicolumn{2}{|c|}{ Innovación en Producto } & \multicolumn{2}{|c|}{ Innovación en Proceso } \\
\hline & \multicolumn{2}{|l|}{ Marginales } & \multirow[t]{2}{*}{$\begin{array}{c}\text { Efectos } \\
\text { marginales }\end{array}$} & \multirow[t]{2}{*}{ P-valor } \\
\hline & & & & \\
\hline País Argentina & 0,1051 & 0,227 & $0,173^{*}$ & $0,075^{*}$ \\
\hline \multicolumn{5}{|l|}{ Variables Internas } \\
\hline Antigüedad & $-0,001$ & 0,359 & $-0,001$ & 0,277 \\
\hline Tamaño & $-0,000$ & 0,922 & $-0,000$ & 0,607 \\
\hline Investigación y desarrollo & $0,226^{* *}$ & $0,000^{* *}$ & $0,161^{* *}$ & $0,019^{* *}$ \\
\hline Calidad & 0,009 & 0,917 & $0,182^{*}$ & $0,064^{*}$ \\
\hline Experiencia del Gerente & $-0,001$ & 0,391 & $-0,003$ & 0,896 \\
\hline Exportaciones Directas & 0,007 & 0,657 & $-0,003$ & 0,224 \\
\hline Exportaciones Indirectas & 0,001 & 0,634 & 0,025 & 0,218 \\
\hline Resultados Anteriores & 0,049 & 0,420 & 0,059 & 0,390 \\
\hline Propietario (Género) & 0,003 & 0,952 & $-0,059$ & 0,379 \\
\hline Capital Extranjero & $-0,000$ & 0,759 & 0,002 & 0,320 \\
\hline Recursos Humanos Calificados & 0,063 & 0,318 & $0,154^{* *}$ & $0,031^{* *}$ \\
\hline \multicolumn{5}{|l|}{ Variables Externas } \\
\hline Tecnología & 0,083 & 0,292 & 0,026 & 0,776 \\
\hline Competencia & $-0,083$ & 0,155 & 0,018 & 0,786 \\
\hline Región (Capital) & 0,046 & 0,430 & $-0,171^{*}$ & $0,010^{*}$ \\
\hline No. observaciones & 273 & & 273 & \\
\hline Log likelihood & -147.35325 & & -170.66685 & \\
\hline Prob > chi2 & $0.0037^{* *}$ & & $0.0013^{* *}$ & \\
\hline
\end{tabular}

**5\% de significancia, ${ }^{*} 10 \%$ de significancia

Fuente: elaboración propia con base en la Enterprise Survey del Banco Mundial, panel 2006-2010.

La tabla 4 muestra los efectos marginales de las variables internas y externas mostrando la comparación de las MIPYMES manufactureras de la Argentina con respecto al Ecuador en la propensión a innovar. En el caso de la Argentina, la investigación y desarrollo incrementa en un porcentaje mayor que el Ecuador ( 24 \% vs. 21,2 \%) la propensión a innovar en el producto; en cuanto a la innova- 
ción en el proceso, en el Ecuador la investigación y desarrollo incrementa en mayor medida que la Argentina en la propensión a innovar (14,5 \% vs. 13,8 \%).

Los resultados de que la I+D es un factor clave para la innovación en las empresas en la Argentina es congruente con los estudios realizados por Chudnovsky et al., 2006; Gonçalves et al., 2008. No obstante, en la innovación en el proceso, aparecen otras variables como los recursos humanos calificados que incrementan en un porcentaje mayor (13,9\%) en el Ecuador que en la Argentina $(13,2 \%)$ la propensión a innovar. La adopción de sistemas de calidad tiene un incremento mayor en el Ecuador (14,5\%) que en la Argentina (13,8\%) respecto de la probabilidad de innovar en el proceso.

A su vez, la región (Buenos Aires, Pichincha) es una variable externa que tiene un efecto inverso a la probabilidad de innovar en el proceso y es significativo para ambos países. Los resultados manifiestan que existen otras variables además de la I+D que pueden influir en la propensión a innovar en el proceso, resultados que se corroboran lo planteado por Arza y López (2010).

Tabla 4. Argentina y Ecuador: análisis comparativo de los efectos marginales de las variables internas y externas de la innovación (2006-2010)

\begin{tabular}{|c|c|c|c|c|}
\hline \multirow[b]{2}{*}{ Variables } & \multicolumn{2}{|c|}{ Innovación en Producto } & \multicolumn{2}{|c|}{ Innovación en Proceso } \\
\hline & $\begin{array}{l}\text { Argentina } \\
\mathrm{df} / \mathrm{dx}\end{array}$ & $\begin{array}{l}\text { Ecuador } \\
\mathrm{df} / \mathrm{dx}\end{array}$ & $\begin{array}{l}\text { Argentina } \\
\text { df/dx }\end{array}$ & $\begin{array}{l}\text { Ecuador } \\
\mathrm{df} / \mathrm{dx}\end{array}$ \\
\hline \multicolumn{5}{|l|}{ Variables Internas } \\
\hline Antigüedad & $-0,001$ & $-0,001$ & $-0,001$ & $-0,001$ \\
\hline Tamaño & $-0,000$ & $-0,000$ & $-0,000$ & $-0,000$ \\
\hline Investigación y desarrollo & $0,240^{* *}$ & $0,212^{* *}$ & $0,138^{* *}$ & $0,145^{* *}$ \\
\hline Calidad & 0,009 & 0,008 & $0,160^{*}$ & $0,168^{*}$ \\
\hline Experiencia del Gerente & $-0,018$ & $-0,001$ & $-0,003$ & $-0,003$ \\
\hline Exportaciones Directas & 0,001 & 0,001 & $-0,021$ & $-0,022$ \\
\hline Exportaciones Indirectas & 0,007 & 0,006 & 0,064 & 0,025 \\
\hline Resultados Anteriores & 0,049 & 0,044 & 0,050 & 0,053 \\
\hline Propietario (Género) & 0,003 & 0,003 & $-0,050$ & $-0,052$ \\
\hline Capital Extranjero & $-0,000$ & $-0,000$ & 0,005 & 0,002 \\
\hline RR HH Calificados & 0,066 & 0,058 & $0,132^{* *}$ & $0,139^{* *}$ \\
\hline \multicolumn{5}{|l|}{ Variables Externas } \\
\hline Tecnología & 0,923 & 0,081 & 0,022 & 0,023 \\
\hline Competencia & $-0,087$ & $-0,077$ & 0,015 & 0,016 \\
\hline Región & 0,047 & 0,041 & $-0,147^{*}$ & $-0,154^{*}$ \\
\hline
\end{tabular}

Fuente: elaboración propia con base en la Enterprise Survey del Banco Mundial, panel 2006-2010 **5\% de significancia, ${ }^{*} 10 \%$ de significancia 


\subsection{Análisis de los efectos de la innovación en el producto y en el proceso (performance)}

\section{Efectos de la innovación en la Argentina y en el Ecuador}

Las tablas 5 y 6 recogen las estimaciones de las variables desempeño cualitativas y cuantitativas de la innovación en el producto y en el proceso de las empresas manufactureras argentinas y ecuatorianas.

Las subtablas A y B evidencian las variables desempeño cualitativas y presentan el p-valor de la regresiones probit en dos etapas, la hipótesis nula rho $=0$ en la regresión biprobit y la regresión probit en una etapa en las últimas filas de las tablas. Se muestran los coeficientes de correlación y la significancia de las variables control del MCO de dos ecuaciones cuando rh0 $=0$ es significante, es decir se acepta la hipótesis de endogeneidad de la innovación. De lo contrario, se presentan los coeficientes de correlación y su significancia de una regresión probit en una etapa cuando tiene significancia estadística.

Las variables desempeño cuantitativas se presentan en las subtablas C y D y presentan el p-valor del MCO en dos etapas, el test Durbin de endogeneidad y el MCO en una etapa en las últimas filas de las tablas. Se muestran los coeficientes de correlación y la significancia de las variables control del MCO de dos ecuaciones cuando el test Durbin es significante es decir se acepta la hipótesis de endogeneidad de la innovación. De lo contrario, se presentan los coeficientes de correlación y su significancia de un MCO de una etapa con su significancia estadística. 


\subsubsection{Efectos de la innovación en la Argentina}

Tabla 5. Estimaciones para la innovación en el producto y en el proceso para la Argentinavariables de la perfomance empresarial cualitativas y cuantitativas

Tabla 5A. Argentina: efectos cualitativos de la innovación en el producto

\begin{tabular}{|c|c|c|c|c|c|c|}
\hline $\begin{array}{c}\text { Efectos/Variables } \\
\text { control }\end{array}$ & $\begin{array}{l}\text { Nuevos } \\
\text { Mercados }\end{array}$ & $\begin{array}{c}\text { Incremento } \\
\text { en el núme- } \\
\text { ro de bienes } \\
\text { ofrecidos } \\
\end{array}$ & $\begin{array}{l}\text { Mejora } \\
\text { de la } \\
\text { calidad }\end{array}$ & $\begin{array}{l}\text { Reducción } \\
\text { de Costos }\end{array}$ & $\begin{array}{l}\text { Reducción } \\
\text { energía }\end{array}$ & $\begin{array}{c}\text { Obtención de } \\
\text { certificaciones } \\
\text { de calidad o } \\
\text { exportación }\end{array}$ \\
\hline $\begin{array}{l}\text { Innovación en el } \\
\text { producto }\end{array}$ & $\begin{array}{r}1,4592^{*} \\
(0,000)\end{array}$ & $\begin{array}{r}2.0368^{*} \\
(0,000)\end{array}$ & $\begin{array}{r}2,2845^{*} \\
(0,000)\end{array}$ & $\begin{aligned} 1,8499^{*} \\
(0,000)\end{aligned}$ & $\begin{array}{l}0,0229 \\
(0,930)\end{array}$ & $\begin{array}{r}1,5676^{*} \\
(0,000)\end{array}$ \\
\hline Antigüedad & $\begin{array}{l}0,0051 \\
(0,106)\end{array}$ & $\begin{array}{r}-0,0160^{* *} \\
(0,067) \\
\end{array}$ & $\begin{array}{r}0,0035^{* *} \\
(0,083)\end{array}$ & $\begin{array}{l}0,0042 \\
(0,219)\end{array}$ & $\begin{array}{l}0,0020 \\
(0,638) \\
\end{array}$ & $\begin{array}{r}-0,0012 \\
(0,743) \\
\end{array}$ \\
\hline Tamaño & $\begin{array}{l}0,0022 \\
(0,143)\end{array}$ & $\begin{array}{r}0,0016 \\
(0,648) \\
\end{array}$ & $\begin{array}{r}-0,0000 \\
(0,969) \\
\end{array}$ & $\begin{array}{l}0,0016 \\
(0,462)\end{array}$ & $\begin{array}{l}0,0009 \\
(0,687)\end{array}$ & $\begin{array}{l}0,0027 \\
(0,165) \\
\end{array}$ \\
\hline $\begin{array}{l}\text { Financiamiento } \\
\text { externo }\end{array}$ & $\begin{array}{l}0,0044 \\
(0,305)\end{array}$ & $\begin{array}{l}0,0129 \\
(0,507)\end{array}$ & $\begin{array}{l}0,0093 \\
(0,171)\end{array}$ & $\begin{array}{l}0,0005 \\
(0,908)\end{array}$ & $\begin{array}{l}-0,0016 \\
(0,814) \\
\end{array}$ & $\begin{array}{l}0,0048 \\
(0,229)\end{array}$ \\
\hline Género del gerente & $\begin{array}{r}-0,0419 \\
(0,919)\end{array}$ & $\begin{array}{r}-0,1063 \\
(0,760)\end{array}$ & $\begin{array}{r}-0,1739 \\
(0,598)\end{array}$ & $\begin{array}{l}0,0770 \\
(0,812)\end{array}$ & $\begin{array}{r}-0,4716 \\
(0,374)\end{array}$ & $\begin{array}{l}-0,2911 \\
(0,158)\end{array}$ \\
\hline Competencia & $\begin{array}{r}-0,0372 \\
(0,291) \\
\end{array}$ & $\begin{array}{l}0,0881 \\
(0,413) \\
\end{array}$ & $\begin{array}{r}-0,0094 \\
(0,825) \\
\end{array}$ & $\begin{array}{r}-0,0056 \\
(0,908) \\
\end{array}$ & $\begin{array}{l}0,0336 \\
(0,550)\end{array}$ & $\begin{array}{r}-0,0450 \\
(0,161) \\
\end{array}$ \\
\hline $\begin{array}{l}\text { Biprobit } \\
\text { Prob }>F\end{array}$ & $0,0000^{*}$ & $0,0025^{*}$ & $0,0000^{*}$ & $0,0000^{*}$ & 0,8050 & $0,0000^{*}$ \\
\hline $\begin{array}{l}\text { Likelihood-ratio } \\
\text { test para rho }=0\end{array}$ & $0,0922^{*}$ & $0,0940^{* *}$ & $0,0198^{*}$ & $0,0979^{*}$ & 0,6182 & $0,0001^{*}$ \\
\hline $\begin{array}{l}\text { Probit } \\
\text { Prob }>F\end{array}$ & $\ldots \ldots$ & $\ldots .$. & $\ldots .$. & $\ldots \ldots$ & 0,9352 & $\ldots \ldots$ \\
\hline
\end{tabular}

Fuente: elaboración propia con base en la Enterprise Survey del Banco Mundial a empresas del sector manufacturero en la Argentina y en el Ecuador, Panel (2006-2010).

*significancia al 0,05, **significancia al 0,10 (p-valores entre paréntesis)

Para la Argentina, la tabla 5A evidencia que los resultados de las regresiones probit (biprobit) en dos etapas son globalmente significativas para las variables de la performance empresarial cualitativas: nuevos mercados, incremento de bienes ofrecidos, mejora de la calidad, reducción de costos y obtención de certificaciones de calidad o exportación. Se rechaza la hipótesis de exogeneidad de la innovación para estas variables dependientes, por lo que estas variables se analizaron con las regresiones probit en dos etapas. La variable reducción de energía no es significativa en la estimación de dos regresiones y no se rechaza la hipótesis nula de exogeneidad de la innovación, por lo que se analiza con una regresión en una etapa, que resultó no significativa globalmente. Los resulta- 
dos muestran un efecto positivo de la variable innovación en el producto de manera individual en nuevos mercados, incremento en el número de bienes ofrecidos, mejora de la calidad y reducción de costos. La única variable control significativa es la antigüedad, con un efecto negativo sobre el incremento en el número de bienes ofrecidos y positivo en la mejora de calidad.

Tabla 5B. Argentina: efectos cualitativos de la innovación en el proceso

\begin{tabular}{|c|c|c|c|c|c|c|}
\hline $\begin{array}{l}\text { Efectos/ } \\
\text { Variables } \\
\text { Control }\end{array}$ & $\begin{array}{l}\text { Nuevos } \\
\text { Mercados }\end{array}$ & $\begin{array}{l}\text { Incremento en } \\
\text { el número de } \\
\text { bienes ofreci- } \\
\text { dos }\end{array}$ & $\begin{array}{l}\text { Mejora } \\
\text { de la } \\
\text { calidad }\end{array}$ & $\begin{array}{c}\text { Reducción } \\
\text { de Costos }\end{array}$ & $\begin{array}{l}\text { Reducción } \\
\text { energía }\end{array}$ & $\begin{array}{c}\text { Obtención de } \\
\text { certificaciones } \\
\text { de calidad o } \\
\text { exportación }\end{array}$ \\
\hline $\begin{array}{l}\text { Innovación en } \\
\text { el proceso }\end{array}$ & $\begin{array}{r}1,2889^{*} \\
(0,058)\end{array}$ & $\begin{array}{r}1,0538^{*} \\
(0,069)\end{array}$ & $\begin{array}{l}0,8691 \\
(0,449)\end{array}$ & $\begin{array}{r}0,5681^{*} \\
(0,002)\end{array}$ & $\begin{array}{l}1,6286 \\
(0,858)\end{array}$ & $\begin{array}{l}1,829^{*} \\
(0,000)\end{array}$ \\
\hline Antigüedad & $\begin{array}{l}0,0069 \\
(0,125)\end{array}$ & $\begin{array}{l}0,0033 \\
(0,415)\end{array}$ & $\begin{array}{l}0,0023 \\
(0,646)\end{array}$ & $\begin{array}{l}0,0013 \\
(0,725)\end{array}$ & $\begin{array}{l}0,0031 \\
(0,541) \\
\end{array}$ & $\begin{array}{r}-0,0016 \\
(0,660)\end{array}$ \\
\hline Tamaño & $\begin{array}{l}0,0018 \\
(0,495)\end{array}$ & $\begin{array}{l}0,0012 \\
(0,640)\end{array}$ & $\begin{array}{l}0,0002 \\
(0,946)\end{array}$ & $\begin{array}{l}0,0232 \\
(0,281)\end{array}$ & $\begin{array}{l}0,0005 \\
(0,860)\end{array}$ & $\begin{array}{l}0,0022 \\
(0,214)\end{array}$ \\
\hline $\begin{array}{l}\text { Financiamiento } \\
\text { externo }\end{array}$ & $\begin{array}{l}0,0082 \\
(0,197)\end{array}$ & $\begin{array}{l}0,0023 \\
(0,699)\end{array}$ & $\begin{array}{l}0,0135 \\
(0,149)\end{array}$ & $\begin{array}{l}0,0023 \\
(0,539)\end{array}$ & $\begin{array}{l}0,0013 \\
(0,851)\end{array}$ & $\begin{array}{l}0,0057 \\
(0,145)\end{array}$ \\
\hline $\begin{array}{l}\text { Género del } \\
\text { gerente }\end{array}$ & $\begin{array}{l}0,2506 \\
(0,588)\end{array}$ & $\begin{array}{l}0,1613 \\
(0,686) \\
\end{array}$ & $\begin{array}{l}0,1361 \\
(0,756)\end{array}$ & $\begin{array}{l}0,0968 \\
(0,801)\end{array}$ & $\begin{array}{r}-0,2579 \\
(0,651)\end{array}$ & $\begin{array}{r}-0,1807^{* *} \\
(0,066) \\
\end{array}$ \\
\hline Competencia & $\begin{array}{l}0,0628 \\
(0,290)\end{array}$ & $\begin{array}{l}0,0141 \\
(0,772)\end{array}$ & $\begin{array}{l}0,0253 \\
(0,669)\end{array}$ & $\begin{array}{r}-0,0283 \\
(0,539)\end{array}$ & $\begin{array}{r}-0,0136 \\
(0,828)\end{array}$ & $\begin{array}{r}-0,0397 \\
(0,129)\end{array}$ \\
\hline $\begin{array}{l}\text { Biprobit } \\
\text { Prob }>\text { F }\end{array}$ & $0,0001^{*}$ & $0,0052^{*}$ & 0,0278 & 0,1153 & 0,2728 & $0,0000^{*}$ \\
\hline $\begin{array}{l}\text { Likelihood- } \\
\text { ratio test para } \\
\text { rho=0 }\end{array}$ & 0,3059 & 0,2942 & 0,7123 & 0,7353 & 0,9385 & $0,0000^{*}$ \\
\hline $\begin{array}{l}\text { Probit } \\
\text { Prob }>F\end{array}$ & 0,6442 & 0,1946 & 0,1946 & $0,0458^{*}$ & 0,5755 & $\ldots .$. \\
\hline
\end{tabular}

Fuente: elaboración propia con base en la Enterprise Survey del Banco Mundial a empresas del sector manufacturero en la Argentina y en el Ecuador, Panel (2006-2010).

*significancia al 0,05, **significancia al 0,10 (p-valores entre paréntesis)

La tabla 5B para la Argentina muestra que se rechaza la hipótesis nula de exogeneidad de la innovación únicamente para la variable obtención de certificaciones de calidad o exportación. En este caso, los resultados de la regresión en dos etapas muestran un efecto positivo de la innovación en proceso, junto con la incidencia negativa de contar con una gerente mujer. Este resultado puede explicarse por diferencias de los sectores productivos en los cuales predomina la contratación de hombres. 
El resto de las variables resultados fueron estimadas mediante regresiones probit simples. Los resultados son globalmente significativos para la reducción en costos, donde la innovación en proceso tiene efecto positivo. Para las restantes variables resultados (nuevos mercados, incremento en el número de bienes ofrecidos, mejora de la calidad y reducción de energía), los resultados son globalmente no significativos.

Tabla 5C. Argentina: efectos cuantitativos de la innovación en el producto

\begin{tabular}{|c|c|c|c|c|c|}
\hline $\begin{array}{c}\text { Efectos/ } \\
\text { Variables Control }\end{array}$ & Ventas & Exportaciones & Empleo & $\begin{array}{l}\text { Fuerza Laboral } \\
\text { Calificada }\end{array}$ & Productividad \\
\hline $\begin{array}{l}\text { Innovación en } \\
\text { producto }\end{array}$ & $\begin{array}{r}-48,2304 \\
(0,135)\end{array}$ & $\begin{array}{r}-0,9877 \\
(0,429)\end{array}$ & $\begin{array}{l}0,0403 \\
(0,380)\end{array}$ & $\begin{array}{l}1,9704 \\
(0,506)\end{array}$ & $\begin{array}{r}-5,962 \\
(0,454)\end{array}$ \\
\hline Antigüedad & $\begin{array}{r}0,0194 \\
(0,843)\end{array}$ & $\begin{array}{l}-0,0178 \\
(0,457)\end{array}$ & $\begin{array}{r}-0,0019^{*} \\
(0,023)\end{array}$ & $\begin{array}{l}-0,0880 \\
(0,454)\end{array}$ & $\begin{array}{r}-0,0237 \\
(0,325)\end{array}$ \\
\hline Tamaño & $\begin{array}{r}0,2249^{*} \\
(0,000)\end{array}$ & $\begin{array}{r}0,0234^{*} \\
(0,074)\end{array}$ & $\begin{array}{l}0,0001 \\
(0,755)\end{array}$ & $\begin{array}{l}0,0080 \\
(0,900)\end{array}$ & $\begin{array}{r}0,0275^{*} \\
(0,056)\end{array}$ \\
\hline $\begin{array}{l}\text { Financiamiento } \\
\text { externo }\end{array}$ & $\begin{array}{l}0,1298 \\
(0,305)\end{array}$ & $\begin{array}{r}-0,0354 \\
(0,327)\end{array}$ & $\begin{array}{l}0,0017 \\
(0,178)\end{array}$ & $\begin{array}{l}-0,1813 \\
(0,191)\end{array}$ & $\begin{array}{l}0,0179 \\
(0,566)\end{array}$ \\
\hline Género del gerente & $\begin{array}{r}-8,7611 \\
(0,245)\end{array}$ & $\begin{array}{l}2,5017 \\
(0,236)\end{array}$ & $\begin{array}{r}0,1895^{*} \\
(0,014)\end{array}$ & $\begin{array}{r}-0,0600 \\
(0,940)\end{array}$ & $\begin{array}{l}-5,962 \\
(0,454)\end{array}$ \\
\hline Competencia & $\begin{array}{r}-3,1835 \\
(0,120)\end{array}$ & $\begin{array}{r}0,6064^{*} \\
(0,036)\end{array}$ & $\begin{array}{l}0,0001 \\
(0,985)\end{array}$ & $\begin{array}{l}0,1507 \\
(0,437)\end{array}$ & $\begin{array}{r}-0,3658 \\
(0,469)\end{array}$ \\
\hline Constante & 43,2240 & $-1,9337$ & 0,1018 & 0,4160 & 8,0957 \\
\hline $\begin{array}{l}\text { MCO de } 2 \text { ecuaciones } \\
\text { Prob }>F\end{array}$ & $0,0007^{*}$ & $0,0067^{*}$ & $0,0525^{*}$ & 0,6560 & 0,4068 \\
\hline $\begin{array}{l}\text { Durbin score } \\
\text { Chi2 }\end{array}$ & $0,0101^{*}$ & 0,8407 & 0,8295 & 0,5158 & 0,3579 \\
\hline $\begin{array}{l}\mathrm{MCO} \\
\text { Prob }>\mathrm{F}\end{array}$ & $\ldots .$. & $0,0136^{*}$ & $0,0230^{*}$ & 0,4630 & 0,2089 \\
\hline
\end{tabular}

Fuente: elaboración propia con base en la Enterprise Survey del Banco Mundial a empresas del sector manufacturero en la Argentina y en el Ecuador, Panel (2006-2010).

*significancia al 0,05, **significancia al 0,10 (p-valores entre paréntesis)

Para la Argentina, la tabla 5C muestra regresiones globalmente significativas en dos etapas (ivregress) para las ventas, las exportaciones y el empleo. En cambio, para la fuerza laboral calificada y la productividad las regresiones en dos etapas no son en conjunto significativas. Como se rechaza la hipótesis de exogeneidad de la innovación solo para las ventas, esta variable dependiente se analizó con la regresión en dos etapas, y el resto de las variables de la performance empresarial se estudian con una regresión en una etapa. Los resultados de las regresiones en 
una etapa son conjuntamente significativos para exportaciones y empleo, y resultan no significativos para fuerza laboral calificada y productividad.

La innovación en producto no presenta efectos significativos para ninguna de las estimaciones. En cuanto a las variables control, el tamaño tiene una incidencia positiva en las ventas y exportaciones, mientras que la antigüedad incide negativamente en el empleo. Contar con una gerente mujer incide positivamente en el empleo, y una elevada competencia tiene un efecto positivo en las exportaciones.

Tabla 5D. Argentina: efectos cuantitativos de la innovación en el proceso

\begin{tabular}{|c|c|c|c|c|c|}
\hline $\begin{array}{c}\text { Efectos/ } \\
\text { Variables control }\end{array}$ & Ventas & Exportaciones & Empleo & $\begin{array}{c}\text { Fuerza Laboral } \\
\text { Calificada }\end{array}$ & Productividad \\
\hline Innovación en proceso & $\begin{array}{l}0,7788 \\
(0,761)\end{array}$ & $\begin{array}{r}8,5474^{* *} \\
(0,069)\end{array}$ & $\begin{array}{r}0,1010^{*} \\
(0,011)\end{array}$ & $\begin{array}{r}-0,9330 \\
(0,498)\end{array}$ & $\begin{array}{l}-0,7403 \\
(0,812)\end{array}$ \\
\hline Antigüedad & $\begin{array}{l}0,0331 \\
(0,564)\end{array}$ & $\begin{array}{l}0,0303 \\
(0,399)\end{array}$ & $\begin{array}{l}-0,0019 \\
(0,021)^{*}\end{array}$ & $\begin{array}{r}-0,1283 \\
(0,158)\end{array}$ & $\begin{array}{r}-0,0191 \\
(0,380)\end{array}$ \\
\hline Tamaño & $\begin{array}{r}0,2262^{*} \\
(0,000)\end{array}$ & $\begin{array}{r}-0,0461^{*} \\
(0,012) \\
\end{array}$ & $\begin{array}{l}0,0000 \\
(0,894) \\
\end{array}$ & $\begin{array}{r}0,0020 \\
(0,702) \\
\end{array}$ & $\begin{array}{l}0,0229 \\
(0,380) \\
\end{array}$ \\
\hline $\begin{array}{l}\text { Financiamiento } \\
\text { externo }\end{array}$ & $\begin{array}{l}0,0841 \\
(0,311)\end{array}$ & $\begin{array}{r}-0,0179 \\
(0,675)\end{array}$ & $\begin{array}{l}0,0019 \\
(0,143)\end{array}$ & $\begin{array}{r}-0,1456 \\
(0,245)\end{array}$ & $\begin{array}{r}0,0120 \\
(0,559\end{array}$ \\
\hline Género del gerente & $\begin{array}{r}-3,3768 \\
(0,500) \\
\end{array}$ & $\begin{array}{l}4,1121 \\
(0,120) \\
\end{array}$ & $\begin{array}{r}0,2019^{*} \\
(0,008)\end{array}$ & $\begin{array}{r}-0,0364 \\
(0,715) \\
\end{array}$ & $\begin{array}{l}2,0772 \\
(0,236) \\
\end{array}$ \\
\hline Competencia & $\begin{array}{l}0,7783 \\
(0,906)\end{array}$ & $\begin{array}{r}0,7339^{*} \\
(0,034)\end{array}$ & $\begin{array}{r}-0,0017 \\
(0,864)\end{array}$ & $\begin{array}{l}0,0354 \\
(0,715)\end{array}$ & $\begin{array}{r}-0,0403 \\
(0,862)\end{array}$ \\
\hline Constante & $-2,4745$ & $-6,7277$ & 0,0838 & 1,6665 & 3,1606 \\
\hline $\begin{array}{l}\text { MCO de } 2 \text { ecuaciones } \\
\text { Prob }>\text { F }\end{array}$ & $0,0000^{*}$ & $0,0080^{*}$ & $0,0539 *$ & 0,6396 & 0,3518 \\
\hline Durbin score (chi2) & 0,7132 & $0,0264^{*}$ & 0,3242 & 0,3110 & 0,8864 \\
\hline $\begin{array}{l}\mathrm{MCO} \\
\text { Prob }>\mathrm{F}\end{array}$ & $0,0000^{*}$ & $\ldots \ldots$ & $0,0023^{*}$ & 0,4552 & 0,2483 \\
\hline
\end{tabular}

Fuente: elaboración propia con base en la Enterprise Survey del Banco Mundial a empresas del sector manufacturero en la Argentina y en el Ecuador, Panel (2006-2010).

*significancia al 0,05, **significancia al 0,10 (p-valores entre paréntesis)

En la tabla 5D, se observan regresiones globalmente significativas en dos etapas (ivregress) para las ventas, las exportaciones y el empleo. En cambio, para la fuerza laboral calificada y la productividad las regresiones en dos etapas no son conjuntamente significativas. Como se rechaza la hipótesis nula de exogeneidad de la innovación para las ventas y las exportaciones, estas variables dependientes se analizaron con la regresión en dos etapas, y el resto de las variables de la performance 
empresarial se analizan con una regresión en una etapa. Los resultados de las regresiones en una etapa son conjuntamente significativos para las variables ventas y empleo, y resultan no significativos para fuerza laboral calificada y productividad.

Los resultados muestran un efecto positivo de la innovación en el proceso en las exportaciones y en el empleo. Con respecto a las variables control, sobre las ventas tiene un efecto positivo el tamaño de la empresa. En las exportaciones incide positivamente la competencia, y de manera negativa el tamaño, y en el empleo incide positivamente el género del gerente y de manera negativa la antigüedad de la empresa.

\subsubsection{Efectos de la innovación en el Ecuador}

Tabla 6. Estimaciones para la innovación en el producto y la innovación en el proceso para el Ecuador- variables de la perfomance empresarial cualitativas y cuantitativas

Tabla 6A. Ecuador: Efectos cualitativos de la innovación en el producto

\begin{tabular}{lcccccc}
\hline $\begin{array}{c}\text { Efectos/ } \\
\text { Variable control }\end{array}$ & $\begin{array}{c}\text { Nuevos } \\
\text { Mercados }\end{array}$ & $\begin{array}{c}\text { Incremento } \\
\text { en el núme- } \\
\text { ro de bienes } \\
\text { ofrecidos }\end{array}$ & $\begin{array}{c}\text { Mejora } \\
\text { de la } \\
\text { calidad }\end{array}$ & $\begin{array}{c}\text { Reducción } \\
\text { de Costos }\end{array}$ & $\begin{array}{c}\text { Reducción } \\
\text { energía }\end{array}$ & $\begin{array}{c}\text { Obtención de } \\
\text { certificaciones } \\
\text { de calidad o } \\
\text { exportación }\end{array}$ \\
\hline $\begin{array}{l}\text { Innovación en el } \\
\text { producto }\end{array}$ & - & - & $-0,5662$ & $1,2630^{*}$ & $1,5714^{*}$ & - \\
\hline Antigüedad & $-0,0098$ & 0,0255 & $-0,0000$ & 0,0020 & $0,0142^{*}$ & 0,0057 \\
& $(0,1688)$ & $(0,471)$ & $(0,997)$ & $(0,865)$ & $(0,058)$ & $(0,726)$ \\
\hline Tamaño & 0,0006 & 0,0958 & $-0,0055$ & $-0,0045$ & 0,0040 & $-0,0016$ \\
\hline Financiamiento & $(0,940)$ & $(0,426)$ & $(0,352)$ & $(0,387)$ & $(0,221)$ & $(0,862)$ \\
externo & $-0,0286$ & $-0,0145$ & $-0,0099$ & $-0,0048$ & 0,0102 & 0,0004 \\
\hline Género del & $(0,138)$ & $(0,205)$ & $(0,939)$ & $(0,730)$ & $(0,404)$ & $(0,970)$ \\
gerente & $-0,4287$ & 0,1116 & 0,6178 & 0,6147 & 0,5457 & 0,1550 \\
\hline Competencia & $(0,595)$ & $(0,889)$ & $(0,315)$ & $(0,238)$ & $(0,289)$ & $(0,852)$ \\
\hline Biprobit & $-0,1017$ & $-0,1135$ & $-0,0099$ & $-0,0590$ & 0,1195 & $-0,1953$ \\
Prob>F & $(0,546)$ & $(0,511)$ & $(0,939)$ & $(0,675)$ & $(0,247)$ & $(0,239)$ \\
\hline $\begin{array}{l}\text { Likelihood-ratio } \\
\text { test para rho=0 }\end{array}$ & 0,8822 & 0,5944 & $0,0002^{*}$ & $0,0112^{*}$ & $0,0728^{*}$ & $\ldots .$. \\
\hline Probit & 0,9850 & 0,1816 & 0,1835 & $0,0244^{*}$ & $0,0313^{*}$ & $\ldots .$. \\
Prob>F & 0,6805 & 0,9239 & 0,2988 & $\ldots .$. & $\ldots .$. & 0,8115 \\
\hline
\end{tabular}

Fuente: elaboración propia con base en la Enterprise Survey del Banco Mundial a empresas del sector manufacturero en la Argentina y en el Ecuador, Panel (2006-2010). *significancia al 0,05, ${ }^{* *}$ significancia al 0,10 (p-valores entre paréntesis).

Nota: En la estimación de las variables resultado Nuevos Mercados, Incremento de Bienes Ofrecidos y Obtención de certificaciones de calidad o exportación, la variable Innovación en el Producto se omitió por su variabilidad y por el tamaño de la muestra.

Escritos Contables y de Administración [vol. 9, n. ${ }^{\circ}$ 1, 2018, págs. 13 a 58] - 44 
Para el Ecuador, la tabla 6A muestra regresiones globalmente significativas en dos etapas (biprobit) para la reducción de energía (consumo de gas, electricidad, etc.) y para la reducción de costos. Dado que se rechaza la hipótesis nula de exogeneidad de la innovación, estas variables se analizaron con la regresión en dos etapas. Los resultados muestran que la innovación en el producto está relacionada positivamente con la reducción de energía y la reducción de costos. En las regresiones restantes (mejora de la calidad), la innovación en el producto no resulta significativa. Con respecto a las variables control, se observa que la antigüedad tiene un efecto positivo en la reducción de energía.

Tabla 6B. Ecuador: efectos cualitativos de la innovación en el proceso

\begin{tabular}{|c|c|c|c|c|c|c|}
\hline $\begin{array}{c}\text { Efectos/Variables } \\
\text { control }\end{array}$ & $\begin{array}{l}\text { Nuevos } \\
\text { Mercados }\end{array}$ & $\begin{array}{l}\text { Incremento } \\
\text { en el núme- } \\
\text { ro de bienes } \\
\text { ofrecidos }\end{array}$ & $\begin{array}{c}\text { Mejora } \\
\text { de la } \\
\text { calidad }\end{array}$ & $\begin{array}{l}\text { Reducción } \\
\text { de Costos }\end{array}$ & $\begin{array}{c}\text { Reducción } \\
\text { energía }\end{array}$ & $\begin{array}{l}\text { Obtención de } \\
\text { certificaciones } \\
\text { de calidad o } \\
\text { exportación }\end{array}$ \\
\hline $\begin{array}{l}\text { Innovación en el } \\
\text { proceso }\end{array}$ & $\begin{array}{r}0,9682^{* *} \\
(0,067)\end{array}$ & $\begin{array}{l}0,2384 \\
(0,614)\end{array}$ & $\begin{array}{l}0,7562 \\
(0,424)\end{array}$ & $\begin{array}{r}-1,7605^{*} \\
(0,001)\end{array}$ & $\begin{array}{r}1,6424^{*} \\
(0,008)\end{array}$ & $\begin{array}{r}-0,7017 \\
(0,252)\end{array}$ \\
\hline Antigüedad & $\begin{array}{r}-0,1251 \\
(0,417) \\
\end{array}$ & $\begin{array}{l}0,0027 \\
(0,626)\end{array}$ & $\begin{array}{l}0,0513 \\
(0,254)\end{array}$ & $\begin{array}{l}0,0022 \\
(0,879)\end{array}$ & $\begin{array}{r}-0,0191 \\
(0,296)\end{array}$ & $\begin{array}{l}0,0097 \\
(0,521)\end{array}$ \\
\hline Tamaño & $\begin{array}{r}0,0024 \\
(0,731) \\
\end{array}$ & $\begin{array}{r}-0,0012 \\
(0,818) \\
\end{array}$ & $\begin{array}{r}-0,0165 \\
(0,244)\end{array}$ & $\begin{array}{r}-0,0016 \\
(0,792) \\
\end{array}$ & $\begin{array}{l}0,0045 \\
(0,524)\end{array}$ & $\begin{array}{r}-0,0022 \\
(0,781)\end{array}$ \\
\hline $\begin{array}{l}\text { Financiamiento } \\
\text { externo }\end{array}$ & $\begin{array}{r}-0,0050 \\
(0,666) \\
\end{array}$ & $\begin{array}{l}0,0140 \\
(0,623)\end{array}$ & $\begin{array}{r}-0,0018 \\
(0,929) \\
\end{array}$ & $\begin{array}{r}0,0032 \\
(0,819)\end{array}$ & $\begin{array}{r}-0,0024 \\
(0,806) \\
\end{array}$ & $\begin{array}{l}0,0077 \\
(0,552) \\
\end{array}$ \\
\hline Género del Gerente & $\begin{array}{r}0,2800 \\
(0,385)\end{array}$ & $\begin{array}{l}-2,6125 \\
(0,673) \\
\end{array}$ & $\ldots \ldots$ & $\begin{array}{l}0,5586 \\
(0,448)\end{array}$ & $\begin{array}{r}0,4438 \\
(0,471) \\
\end{array}$ & $\begin{array}{r}-0,0124 \\
(0,987) \\
\end{array}$ \\
\hline Competencia & $\begin{array}{l}0,1184 \\
(0,536)\end{array}$ & $\begin{array}{l}0,0405 \\
(0,792)\end{array}$ & $\begin{array}{l}0,1042 \\
(0,698)\end{array}$ & $\begin{array}{r}-0,0771 \\
(0,672)\end{array}$ & $\begin{array}{l}0,1745 \\
(0,247)\end{array}$ & $\begin{array}{r}-0,2430 \\
(0,122)\end{array}$ \\
\hline $\begin{array}{l}\text { Biprobit } \\
\text { Prob }>F\end{array}$ & 0,1089 & 0,3459 & $0,0054^{*}$ & $0,0292^{*}$ & $0,0152^{*}$ & $0,0779 *$ \\
\hline $\begin{array}{l}\text { Likelihood-ratio } \\
\text { test para rho=0 }\end{array}$ & $0,0129^{*}$ & $0,0029^{*}$ & 0,4471 & $0,0398^{*}$ & 0,3463 & 0,1099 \\
\hline $\begin{array}{l}\text { Probit } \\
\text { Prob }>\text { F }\end{array}$ & $\ldots \ldots$ & $\ldots \ldots$ & 0,6241 & $\ldots \ldots$ & 0,1136 & 0,6254 \\
\hline
\end{tabular}

Fuente: elaboración propia con base en la Enterprise Survey del Banco Mundial a empresas del sector manufacturero en la Argentina y en el Ecuador, Panel (2006-2010). *significancia al 0,05, **significancia al 0,10 (p-valores entre paréntesis).

Nota: En la estimación de las variables resultado Mejora de la Calidad se omitió la variable género del gerente por su variabilidad y por el tamaño de la muestra.

En relación con los efectos cualitativos de la innovación en el proceso para el Ecuador, la tabla 6B muestra que se rechaza la hipótesis de exogeneidad para las variables nuevos mercados, incremento de bienes ofrecidos y reducción de 
costos, siendo esta última la única regresión globalmente significativa en dos etapas. En este caso, se observa un efecto negativo de la innovación en proceso sobre la Reducción de Costos. Las restantes variables resultado (Mejora de la Calidad, Reducción de Energía y la Obtención de Certificaciones de Calidad o Exportación) se estiman en una etapa y no resultaron globalmente significativas.

Tabla 6C. Ecuador Efectos cuantitativos de la innovación en el producto

\begin{tabular}{lrrrrr}
\hline $\begin{array}{c}\text { Efectos/Variables } \\
\text { control }\end{array}$ & \multicolumn{1}{c}{ Ventas } & Exportaciones & Empleo & $\begin{array}{r}\text { Fuerza Labo- } \\
\text { ral Calificada }\end{array}$ & Productividad \\
\hline Innovación en el & $-59,7717$ & $-13,4531$ & 0,4600 & 2,0198 & $-1,4287$ \\
producto & $(0,158)$ & $(0,693)$ & $(0,522)$ & $(0,423)$ & $(0,535)$ \\
\hline \multirow{2}{*}{ Antigüedad } & 0,1112 & 0,0534 & $-0,0012$ & $-0,0078$ & $-0,0029$ \\
& $(0,750)$ & $(0,820)$ & $(0,819)$ & $(0,714)$ & $(0,878)$ \\
\hline Tamaño & $0,2687^{*}$ & $-0,0360$ & $-0,0002$ & $-0,0063$ & $0,0146^{*}$ \\
& $(0,048)$ & $(0,688)$ & $(0,839)$ & $(0,522)$ & $(0,048)$ \\
\hline Financiamiento & 0,2869 & 0,1460 & $-0,0055$ & 0,0008 & $-0,0035$ \\
externo & $(0,479)$ & $(0,632)$ & $(0,393)$ & $(0,975)$ & $(0,873)$ \\
\hline Características del & 10,2124 & 3,4186 & 0,0758 & 0,8787 & 0,1471 \\
propietario & $(0,416)$ & $(0,441)$ & $(0,703)$ & $(0,306)$ & $(0,830)$ \\
\hline Competencia & 1,4728 & $-0,2010$ & $-0,0339$ & $-0,0606$ & $-0,0508$ \\
& $(0,604)$ & $(0,854)$ & $(0,819)$ & $(0,766)$ & $(0,742)$ \\
\hline Constante & 22,6016 & 6,3627 & $-0,0579$ & 1,2237 & 1,8937 \\
\hline MCO de 2 ecuaciones & $0,0124^{*}$ & 0,9753 & 0,8310 & 0,5929 & 0,1371 \\
Prob>F & & & & & \\
\hline Durbin score & $0,0763^{* *}$ & 0,7200 & 0,3317 & 0,6097 & 0,3680 \\
Chi2 & & & & 0,6029 & 0,6371 \\
\hline MCO & $\ldots .$. & 0,8873 & 0,4543 & & \\
Prob>F & & & & & \\
\hline
\end{tabular}

Fuente: elaboración propia con base en la Enterprise Survey del Banco Mundial a empresas del sector manufacturero en la Argentina y en el Ecuador, Panel (2006-2010). *significancia al 0,05, **significancia al 0,10 (p-valores entre paréntesis)

En la tabla 6C, para el Ecuador la innovación en el producto muestra regresiones de dos etapas (ivregress) con una significancia global únicamente en las ventas, donde se rechaza además la exogeneidad de la variable innovación. Sin embargo, la innovación no resulta individualmente significativa. La variable control con relevancia es el tamaño de la empresa, con una relación positiva con las ventas. 
Las variables exportaciones, empleo, fuerza laboral calificada y productividad no resultaron significantes en las regresiones de dos etapas y no se rechaza la hipótesis de exogeneidad de la innovación, por lo que estas variables se estudiaron en una regresión en una etapa. Las regresiones así estimadas no resultaron conjuntamente significativas.

Tabla 6D. Ecuador Efectos cuantitativos de la innovación en el proceso

\begin{tabular}{lrrrrr}
\hline $\begin{array}{l}\text { Efectos/Variables } \\
\text { control }\end{array}$ & \multicolumn{1}{l}{ Ventas } & Exportaciones & Empleo & $\begin{array}{r}\text { Fuerza Laboral } \\
\text { Calificada }\end{array}$ & Productividad \\
\hline \multirow{2}{*}{ Innovación en proceso } & 9,6015 & $-14,5454$ & 0,0424 & 2,0198 & 0,6982 \\
& $(0,128)$ & $(0,322)$ & $(0,912)$ & $(0,423)$ & $(0,700)$ \\
\hline \multirow{2}{*}{ Antigüedad } & $-0,1866$ & $-0,0935$ & 0,0018 & 0,8787 & $-0,0083$ \\
& $(0,212)$ & $(0,363)$ & $(0,539)$ & $(0,306)$ & $(0,551)$ \\
\hline \multirow{2}{*}{ Tamaño } & $0,3406^{*}$ & 0,0132 & $-0,0003$ & $-0,0063$ & $0,0157^{*}$ \\
& $(0,000)$ & $(0,762)$ & $(0,591)$ & $(0,522)$ & $(0,014)$ \\
\hline Financiamiento & 0,1876 & 0,1439 & $-0,0022$ & 0,0080 & $-0,0192$ \\
externo & $(0,176)$ & $(0,319)$ & $(0,531)$ & $(0,975)$ & $(0,310)$ \\
\hline Características del & 12,088 & 4,8440 & $-0,0004$ & 0,8787 & 0,1998 \\
propietario & $(0,125)$ & $(0,278)$ & $(0,997)$ & $(0,306)$ & $(0,733)$ \\
\hline \multirow{2}{*}{ Competencia } & 2,5704 & $-0,8391$ & $-0,0349$ & $-0,0606$ & $-0,0015$ \\
Constante & $(0,121)$ & $(0,394)$ & $(0,223)$ & $(0,766)$ & $(0,993)$ \\
\hline MCO de 2 ecuaciones & $-13,9932$ & 8,0228 & 0,1318 & 1,2237 & 0,7671 \\
Prob>F & $0,0000^{*}$ & 0,9055 & 0,6378 & 0,5929 & $0,0499^{*}$ \\
\hline Durbin score & & & & & 0,8264 \\
Chi2 Prob>F & 0,6839 & 0,4563 & 0,7619 & 0,6097 & \\
\hline MCO & & & & & 0,5103 \\
Prob>F & $0,0002^{*}$ & 0,8404 & 0,3057 & & 0,3438 \\
\hline
\end{tabular}

Fuente: elaboración propia con base en la Enterprise Survey del Banco Mundial a empresas del sector manufacturero en la Argentina y en el Ecuador, Panel (2006-2010). *significancia al 0,05, ${ }^{* *}$ significancia al 0,10 (p-valores entre paréntesis)

Los resultados de la tabla $6 \mathrm{D}$ indican que en todos los casos analizados no se rechaza la hipótesis de exogeneidad de la innovación, por lo cual se presentan las estimaciones en una etapa. La única regresión globalmente significativa tiene como variable dependiente a las ventas, encontrándose que la innovación en el proceso no resulta significativa, mientras que el tamaño tiene un efecto positivo.

En la tabla 7 se resumen los resultados de las estimaciones que muestran un efecto positivo de la innovación tanto en el producto como en el proceso 
sobre las variables desempeño, así como un efecto negativo de la innovación en el proceso.

Tabla 7. Argentina y Ecuador: comparación de efectos de la innovación del producto y proceso

\begin{tabular}{|c|c|c|}
\hline \multirow{9}{*}{ Innovación en producto } & Argentina & Ecuador \\
\hline & \multicolumn{2}{|c|}{ Con innovación como variable endógena (biprobit/ivregress) } \\
\hline & Nuevos mercados. & \\
\hline & Incremento en el número de bienes & \\
\hline & ofrecidos. & Reducción energía. \\
\hline & Mejora de la calidad. & Reducción de costos. \\
\hline & $\begin{array}{l}\text { Obtención de certificaciones de } \\
\text { calidad o exportación. }\end{array}$ & \\
\hline & \multicolumn{2}{|c|}{ Con innovación como variable exógena (probit/regress) } \\
\hline & \multicolumn{2}{|l|}{ Reducción de Costos } \\
\hline \multirow{6}{*}{ Innovación en proceso } & \multicolumn{2}{|c|}{ Con innovación como variable endógena (biprobit/ivregress) } \\
\hline & $\begin{array}{l}\text { Obtención de certificaciones de } \\
\text { calidad o de exportación }\end{array}$ & Reducción de costos (-) \\
\hline & Exportaciones & \\
\hline & \multicolumn{2}{|c|}{ Con innovación como variable exógena (probit/regress) } \\
\hline & Reducción de costos & \\
\hline & Empleo & \\
\hline
\end{tabular}

Fuente: elaboración propia con base en tablas 5 y 6.

\section{Consideraciones finales}

El análisis realizado en este resumen muestra la existencia de diferencias y convergencias en las actividades de innovación que realizan las micro, pequeñas y medianas empresas del sector manufacturero argentino y ecuatoriano que formaron parte del estudio, a través del mejor uso de la información cualitativa y cuantitativa disponible en la Enterprise Survey del Banco Mundial de los años 2006 y 2010. Ambos sectores manufactureros están orientados a realizar actividades de innovación, sin embargo tienen un bajo esfuerzo en el gasto en I+D. En referencia a las diferencias, los empresarios del sector manufacturero argentino tienen una apreciación de su propensión a innovar mayor que el ecuatoriano considerando la tipología de la innovación del producto y la innovación del proceso planteada por la OCDE (2005), lo que evidencia que la Argentina tiene un perfil más alto en innovación en el producto y en el proce- 
so que el Ecuador en este análisis de comparabilidad. Así también, esta comparación plantea que la innovación en el producto es mayor a la innovación del proceso en el sector manufacturero en ambos países, lo que lleva a pensar que innovar en el proceso conlleva una mayor inversión en maquinarias, equipos, programas que hace que el empresario decida innovar en el producto y en menor medida en los procesos. En el caso del Ecuador, el análisis evidencia una menor propensión a innovar en el producto y en el proceso comparado con la Argentina y se sugiere que puede deberse a los obstáculos que el empresario encuentra para innovar y a los escasos instrumentos de la política de ciencia, tecnología e innovación en el país.

Los resultados evidenciaron que además de las actividades de I+D, los recursos humanos calificados y la calidad surgen como variables internas latentes de la innovación en el proceso, por lo que las políticas empresariales deberían enmarcarse en el fortalecimiento de estas actividades para la mejora de los resultados y como aspectos que influencian la innovación en las empresas.

En lo que respecta a los efectos de la innovación, se evidencian impactos diferentes con su control endógeno. En la innovación en el producto en el sector manufacturero del Ecuador se manifiesta una significancia positiva en la reducción del consumo de energía (gas, electricidad, etc.) y en el sector manufacturero de la Argentina un efecto positivo en la apertura de nuevos mercados, la mejora de la calidad, el incremento en el número de bienes ofrecidos y la obtención de certificaciones de calidad o de exportación. El efecto semejante positivo de la innovación del producto que se observa en ambos países es la reducción de costos con un control endógeno de la innovación solamente en el Ecuador.

Con referencia a la innovación en el proceso tras controlar su endogeneidad, el sector manufacturero del Ecuador muestra un efecto positivo en la reducción de energía (gas, electricidad, etc.); en la Argentina, la innovación en el proceso genera un efecto positivo en la obtención de certificaciones de calidad o exportación y en las exportaciones y en el empleo, a la vez que genera un efecto positivo sin controlar su endogeneidad. Con respecto a los impactos similares, en ambos sectores se observa a la reducción de costos como un efecto, sin embargo en el Ecuador la significancia de la innovación en el proceso es negativa con un modelo biprobit en este impacto y en la Argentina esta varia- 
ble desempeño es significativa y positiva con un modelo probit simple. Las actividades de innovación realizadas por las MIPYMES manufactureras no presentaron efectos significativos sobre las ventas, la productividad y la fuerza laboral calificada en ambos países. Las variables control que tuvieron relevancia en el estudio fueron el tamaño de la empresa, la antigüedad, el género del gerente y el grado de competencia.

En comparación con el Ecuador, la Argentina presenta mayores impactos en la performance empresarial. Esta evidencia revela que las empresas manufactureras argentinas poseen mayores resultados de su actividad innovadora posiblemente por un esfuerzo mayor en la inversión en ciencia, tecnología e innovación y por un sistema de innovación y tecnología más consolidado, con la presencia de instituciones y fondos e incentivos que dinamiza la política industrial y tecnológica en el país. De esta manera, se sugiere que el Ecuador promueva y facilite los procesos de ciencia, tecnología e innovación mediante el apoyo al financiamiento, una política fiscal con incentivos, la inversión en I+D $+\mathrm{i}$, la creación de instrumentos en política de CTI, entre otros factores para la consecución de mayores impactos de la innovación empresarial.

Respecto de las limitaciones del estudio, si bien es amplio el abanico de estudios relativos a la innovación, trabajar con los datos de la encuesta empresarial del Banco Mundial para ambos países aportó una limitación al análisis por la información reducida de la muestra que presenta el Ecuador. Es importante aclarar que el tamaño de la muestra en el Ecuador pudo ser una limitación, sin embargo la información del Banco Mundial permitió generar un aporte a la comparabilidad de un sector industrial del Ecuador con otro país de la región como la Argentina.

De hecho, también resultaría interesante luego de haber analizado los inputs, los outputs y sus efectos en la performance empresarial de las empresas manufactureras entre el Ecuador y la Argentina, identificar los obstáculos que impiden el proceso innovador en estos países con diferente perfil de CTI, estudio que puede contribuir a la formulación de políticas públicas para la cooperación en el desarrollo de la innovación y al planteamiento de líneas de actuación en torno a la mejora de la competitividad empresarial al interior de cada país. 


\section{Referencias bibliográficas}

Adeyeye, A., Jegede, O., Oluwadare, A. y Aremu, F. (2015). Micro-level determinants of innovation: analysis of the Nigerian manufacturing sector. Innovation and Development, 1-14.

Aghion, P., Howitt, P., Brant-Collet, M. y García-Peñalosa, C. (1988). Endogenous growth theory. Cambridge MA: The MIT Press, 356 p.

Álvarez, E. y García, W. (2012). Determinantes de la innovación: evidencia en el sector manufacturero de Bogotá. Semestre Económico, 15(32), 129-160.

Antonelli, C. (2017). Endogenous innovation: the creative response. Economics of Innovation and New Technology, 26(8), 689-718.

Arza, V. y López, A. (2010). Innovation and productivity in the argentine manufacturing sector. IDB Working Papers Series No. IDB-WP-187, (p. 36).

Consultado el 15 de julio de 2015. Disponible en:

http://www.iadb.org/res/publications/pubfiles/pubIDB-WP-187.pdf

Astudillo, S. y Briozzo, A. (2015). Factores determinantes de la innovación en las MIPYMES manufactureras de la Argentina y el Ecuador. FAEDPYME INTERNATIONAL REVIEW, 4(7), 53-65.

Avermaete, T., Viaene, J., Morgan, E. J., Pitts, E., Crawford, N. y Mahon, D. (2004). Determinants of product and process innovation in small food manufacturing firms. Trends in Food Science \& Technology, 15(10), 474-483.

Banco Mundial. Datos (2010). Consultado el 14 de septiembre de 2014. Disponible en: https:/ / datos.bancomundial.org/

Barletta, F. y Suárez, D. (2015). Encuestas de innovación en Iberoamérica: Avances en la medición y desafíos futuros. El estado de la ciencia 2014. Buenos Aires, Argentina: RICYT, OEI.

Bartels, F., Hinrich Voss H., Lederer, S. y Bachtrog, C. (2012). Determinants of National Innovation Systems: Policy implications for developing countries. Innovation: Management, Policy \& Practice, 14:1, 2-18

Bartoloni, E. (2013). Capital structure and innovation: causality and determinants. Empirica, 40(1), 111-151.

Baum, C. (2006). An Introduction to Modern Econometrics Using Stata. United States of America: Stata Corp LP, 349 p. 
Bayraktar, C. A., Hancerliogullari, G., Cetinguc, B. y Calisir, F. (2017). Competitive strategies, innovation, and firm performance: an empirical study in a developing economy environment. Technology Analysis \& Strategic Management, 29(1), 38-52.

Becheikh, N., Landry, R. y Amara, N. (2006). Lessons from innovation empirical studies in the manufacturing sector: A systematic review of the literature from 1993-2003. Technovation, 26(5), 644-664.

Božić, L. y Radas, S. (2006). The effects of innovation activities in SMEs in the Republic of Croatia. Croatian Economic Survey, (8), 33-52.

Brown, F. y Guzmán, A. (2014). Innovation and Productivity across Mexican Manufacturing Firms. Journal of Technology Management $\mathcal{E}$ Innovation, 9(4), 36-52.

Busom, I. y Vélez-Ospina, J., (2017). Innovation, Public Support, and Productivity in Colombia. A Cross-industry Comparison. World Development, 99, issue C, p. 75-94.

Caldera, A. (2010). Innovation and exporting: evidence from Spanish manufacturing firms. Review of World Economics, 146(4), 657-689.

Cameron, C. Triverdi, P.K. (2009). Microeconometrics using Stata, TX, United States of America: Stata Press College Station, 706p.

Chudnovsky, D., López, A. y Pupato, G. (2006). Innovation and productivity in developing countries: A study of Argentine manufacturing firms's behavior (1992-2001). Research Policy, 35(2), 266-288.

Crépon, B., Duguet, E. y Mairesse, J. (1998). Research, Innovation and Productivity: An Econometric Analysis at the Firm Level. Economics of Innovation and New Technology, 7(2), 115-158.

Crespi, G., Tacsir, E. y Vargas, F. (2016) Innovation Dynamics and Productivity: Evidence for Latin America. En: Grazzi, M., Pietrobelli, C. (eds.). Firm Innovation and Productivity in Latin America and the Caribbean. Palgrave Macmillan, Inter-American Development Bank: New York, p. 37-72.

De Fuentes, C., Dutrénit, G., Santiago, F. y Gras, N. (2015). Determinants of Innovation and Productivity in the Service Sector in Mexico. Emerging Markets Finance and Trade, 51(3), 578-592. 
De Jong, J. P. y Vermeulen, P. A. (2006). Determinants of product innovation in small firms a comparison across industries. International Small Business Journal, 24(6), 587-609.

Dini, M., Rovira, S. y Stumpo, G. (2014). Políticas de innovación para pymes en América Latina. Santiago de Chile: CEPAL- Naciones Unidas. Consultado el 16 de julio de 2015. Disponible en:

http://www.cepal.org/es/publicaciones/37352-una-promesa-y-unsuspirar-politicas-de-innovacion-para-pymes-en-america-latina

Dosi, G., Freeman, C., Nelson, R., Silverberg, G. y Soete, L. (1988). Technical Change and Economic Theory. London: Pinter.

Drucker, P. F. (1986). Innovation and Entrepreneurship: Practice and Principles. New York: Harper \& Row.

Durbin, J. (1954). Errors in Variables. Revue De L'Institut International De Statistique / Review of the International Statistical Institute, 22(1/3), 23-32.

Dutrénit, G., Jasso J. y Villavicencio, D. (2007). Globalización, acumulación de capacidades e innovación: Los desafíos para las empresas, localidades y países. México: Fondo de Cultura Económica, Organización de Estados Iberoamericanos para la Educación, la Ciencia y la Cultura, 483 p.

Dutrénit, G. y Katz, J. (2005). Introduction: Innovation, growth and development in Latin-America: Stylized facts and a policy agenda. Innovation, 7(2-3), p. 105-130.

Dutrénit, G., Puchet Anyul, M., Sanz-Menéndez, L., Teubal, M. y Vera-Cruz, A. (2010). Un modelo de política para fomentar la co-evolución de la ciencia y la tecnología con la innovación: el caso mexicano. En: Bracamonte, A., Contreras, O. (eds.) Ciencia, Tecnología e Innovación para el desarrollo económico. México: El Colegio de Sonora/COECyT, p.103-151

Dutrénit, G., Puchet, M. y Teubal, M. (2016). Un modelo apreciativo de coevolución entre ciencia, tecnología e innovación para analizar la dinámica del desarrollo. En: Erbes, A., Suárez, D. (eds.). Repensando el desarrollo: una discusión desde los sistemas de innovación - Buenos Aires: Universidad Nacional de General Sarmiento, p.103-142.

Edquist, C. (2001). Systems of Innovation for Development (SID). Background Paper for Chapter I: "Competitiveness, Innovation and Learning: Analytical Framework" for World Industrial Development Report - UNIDO. 
Enterprise Survey-Raw Data. Banco Mundial (2006-2010). Consultado el 12 de noviembre de 2013. Disponible en: http:/ / espanol.enterprisesurveys.org/

Evangelista, R. y Vezzani, A. (2010). The economic impact of technological and organizational innovations. A firm-level analysis. Research Policy, 39(10), 1253-1263.

Fagerberg, J. (2013). Innovation - a New Guide. Working Papers on Innovation Studies. Centre for Technology, Innovation and Culture, University of Oslo, p. 1-46.

Fagerberg, J. (2004). Innovation a guide to the literature. En: Fagerberg, J., Mowery, David, C., Nelson, R. The Oxford Handbook of Innovation. New York: Oxford University Press, p. 1-27.

François, J. P., Favre, F. y Negassi, S. (2002). Competence and Organization: Two Drivers of Innovation. Economics of Innovation and New Technology, 11(3), 249-270.

Freeman, C. (1975). Teoría Económica de la Innovación Industrial. Madrid: Alianza Editorial.

Fundación Observatorio Pyme (2013). Definiciones de PyME en Argentina y el resto del mundo. Consultado el 15 de julio de 2015. Disponible en: http://www.observatoriopyme.org.ar/project/definiciones-de-pyme-enargentina-y-el- resto-del-mundo-abril-2013/

Gálvez Albarracín, E. J. y García Pérez de Lema, D. (2012). Impacto de la innovación sobre el rendimiento de la mipyme: Un estudio empírico en Colombia. Estudios Gerenciales, 28(122), 11-27.

Goedhuys, M. y Veugelers, R. (2012). Innovation strategies, process and product innovations and growth: Firm-level evidence from Brazil. SI: Firm Dynamics and SI: Globelics Conference, 23(4), 516-529.

Gómez-Vieites, A. y Calvo J.L. (2011). Innovación: Factor clave del éxito empresarial. Bogotá, Colombia: Ecoe Ediciones.

Gonçalves, E., Lemos, M. B. y De Negri, J. (2008). Determinants of technological innovation in Argentina and Brazil. Cepal Review, (94), 71-95.

Grazzi, M. y Jung, J. (2016). Information and Communication Technologies, Innovation, and Productivity: Evidence from Firms in Latin America and the Caribbean. En: Grazzi, M., Pietrobelli, C. (eds.). Firm Innovation and 
Productivity in Latin America and the Caribbean. Palgrave Macmillan, InterAmerican Development Bank: New York, p.103-136.

Grossman, G. M., Helpman, E. (1993). Innovation and growth in the global economy. Cambridge MA: The MIT Press.

Gujarati, D. y Porter, D. (2009). Econometría. México: McGraw-Hill, 1003 p.

Gunday, G., Ulusoy, G., Kilic, K., Alpkan, L. (2011). Effects of innovation types on firm performance. International Journal of Production Economics, 133(2), 662-676.

Hassan, M. U., Shaukat, S., Nawaz, M. S. y Naz, S. (2013). Effects of innovation types on firm performance: An empirical study on Pakistan's manufacturing sector. Journal of Commerce and Social Sciences, 7(2), 243-262.

Jung, D. I., Chow, C. y Wu, A. (2003). The role of transformational leadership in enhancing organizational innovation: Hypotheses and some preliminary findings. Leading for Innovation, 14(4-5), 525-544.

Kline, S. y Rosenberg, G. (1986). An Overview of Innovation. En: Landau, R., Rosenberg, N. (eds.). The positive sum strategy: harnessing technology for economic growth, Washington, DC, National Academy Press, p. 275-305.

Janz, N.; Lööf, H. y Peters, B. (2004). Firm Level Innovation and Productivity - Is there a Common Story across Countries? (Working Paper Series in Economics and Institutions of Innovation No. 24). En: Royal Institute of Technology, CESIS - Centre of Excellence for Science and Innovation Studies, p. 1-23.

Klomp, L. y Van Leeuwen, G. (2001). Linking innovation and firm performance: a new approach. International Journal of the Economics of Business, 8(3), 343-364.

Laforet, S. (2008). Size, strategic, and market orientation effects on innovation. Journal of Business Research, 61(7), 753-764.

Landry, R., Amara, N. y Lamari, M. (2002). Does social capital determine innovation? To what extent? Technological Forecasting and Social Change, 69(7), 681-701.

Lee, J. (1995). Small firms' innovation in two technological settings. Research Policy, 24(3), 391-401.

Lööf, H., Heshmati, A., Asplund, R. y Nåås, S.-O. (2001). Innovation and performance in manufacturing industries: A comparison of the Nordic Countries. SSE/EFI Working Paper Series in Economics and Finance. 
Consultado el 15 de Septiembre de 2016. Disponible en: http:/ / core.kmi.open.ac.uk/download/pdf/7097896.pdf

López-Mielgo, N., Montes-Peón, J. M. y Vázquez-Ordás, C. (2012). ¿Qué necesita una empresa para innovar? Investigación, experiencia y persistencia. Revista Europea de Dirección y Economía de la Empresa, 21(3), 266-281.

Love, J. H. y Roper, S. (2015). SME innovation, exporting and growth: A review of existing evidence. International Small Business Journal, 33(1), 28-48.

Lundvall, B. Å. (2009). Sistemas Nacionales de Innovación: Hacia una teoría de la Innovación y el Aprendizaje por Interacción. Provincia de Buenos Aires, Argentina: UNSAM EDITA.

Lundvall, B.Å. (1992). National System of Innovation: Towards a Theory of Innovation and Interactive Learning. Pinter: London.

Madeira Silva, M., Simões, J., Sousa, G., Moreira, J. y Wagner Mainardes, E. (2014). Determinants of innovation capacity: Empirical evidence from services firms. Innovation, 16(3), 404-416.

Mairesse, J. y Mohnen, P. (2010). Using Innovations Surveys for Econometric Analysis (Working Paper No. 15857). National Bureau of Economic Research, 40 p. Consultado el 20 de agosto de 2014. Disponible en: http://www.nber.org/papers/w15857.

Malerba, F. y Orsenigo, L. (1996). Schumpeterian patterns of innovation are technology-specific. Research Policy, 25(3), 451-478.

Ma, C. y Liu, Z. (2017). Effects of M\&As on innovation performance: empirical evidence from Chinese listed manufacturing enterprises. Technology Analysis \& Strategic Management, 29(8), 960-972.

Martins, I., Goméz-Araujo, E. y Vaillant, Y. (2015). Mutual effects between Innovation commitment and exports: Evidence from the owner-manager in Colombia. Journal of Technology Management $\mathcal{E}$ Innovation, 10(1), 103-116.

Metcalfe, J. S. (1998). Evolutionary Economics and Creative Destruction. London, England: Psychology Press.

Milesi, D., Petelski., N. y Verre, V., (2011). The determinants of innovation: Evidence from Argentine Manufacturing firms. Provincia de Buenos Aires, Argentina: Universidad General Sarmiento, Instituto de la Industria.

Nelson, R. y Winter, S. (1982). An evolutionary theory of economic change. Cambridge, Massachusetts: Harvard University Press, 452p. 
OCDE-Organización de Cooperación y Desarrollo Económicos (2005). Manual de Oslo: Guía para la recogida e interpretación de datos sobre innovación. $3^{\mathrm{a}}$ ed., Oficina de Estadísticas de las Comunidades Europeas, París: OCDE, 194p.

Peters, B. (2008). Innovation and firm performance: An empirical investigation for German firms (Vol. 38). New York: Physica-Verlag, Heidelberg.

Pianta, M. (2003). Innovation and employment. En: Fagerberg, J., Mowery, D., Nelson, R. (eds.). Handbook of Innovation, The Oxford University Press (forth), 1-33 pp.

Porter, M. (1990). Competitive Advantage of Nations, with a new introduction. United States of America. New York: Free Press, 1035p.

Prajogo, D. I. y Sohal, A. S. (2003). The relationship between TQM practices, quality performance, and innovation performance: An empirical examination. International Journal of Quality \& Reliability Management, 20(8), 901-918.

Raymond, L. y St-Pierre, J. (2010). R\&D as a determinant of innovation in manufacturing SMEs: An attempt at empirical clarification. Technovation, 30(1), 48-56.

Registro OficialTM (2010). Reglamento al código orgánico de la producción, comercio e inversiones. Administración del Sr. Econ. Rafael Correa Delgado, Presidente Constitucional de la República del Ecuador. (pp. 353). Consultado el 15 de agosto de 2014. Disponible en: http://www.produccion.gob.ec/wpcontent/uploads/downloads/ 2013/01/ReglamentosCodigoProduccion.pdf.

Reichert, F. M. y Zawislak, P. A. (2014). Technological capability and firm performance. Journal of Technology Management E Innovation, 9(4), 20-35.

Rhee, J., Park, T. y Lee, D. H. (2010). Drivers of innovativeness and performance for innovative SMEs in South Korea: Mediation of learning orientation. Technovation, 30(1), 65-75.

Romijn, H. y Albaladejo, M. (2002). Determinants of innovation capability in small electronics and software firms in southeast England. Research Policy, 31(7), 1053-1067, $235 \mathrm{p}$.

Roper, S. y Arvanitis, S. (2012). From knowledge to added value: A comparative, panel-data analysis of the innovation value chain in Irish and Swiss manufacturing firms, Research Policy, 41(6), 1093-1106.

Rosegger, G. (1996). The economics of production and innovation. An Industrial Perspective. Third Edition, Oxford, London: Pergamon Press. 
Sandven, T. y Smith, K. H. (2000). Innovation and economic performance at the enterprise level. France, 23-24/11/2000.Conference innovation and enterprise creation: Statistics and indicators, Norway. Consultado el 20 de Septiembre de 2016. Disponible en: http://www.nifu.no/files/2012/11/STEPrapport10-2000.pdf

Schumpeter, J. A. (1912). The theory of economic development. Harvard Economic Studies, 46, $255 \mathrm{p}$.

Schumpeter, J. A. (1934). The theory of economic development. Cambridge: Harvard University Press, 255p.

Sørensen, J. B., Stuart, T. E. (2000). Aging, obsolescence, and organizational innovation. Administrative Science Quarterly, 45(1), 81-112.

Sternberg, R. y Arndt, O. (2001). The firm or the region: what determines the innovation behavior of European firms?. Economic Geography, 77(4), 364-382.

Suárez, D. (2013). Innovativa strategies in unstable environments. The Argentinean case. Aalborg University. PhD Thesis. Consultado el 2 de enero de 2016. Disponible en:

http://www.ungs.edu.ar/ms_idei/publicaciones/index.php/innovativestrategies-in-unstable-environments-the-case-of-argentinean-firms/

Terziovski, M. y Guerrero, J. L. (2014). ISO 9000 quality system certification and its impact on product and process innovation performance. International Journal of Production Economics, 158 (12), 197-207.

Thai Hoang, D., Igel, B. y Laosirihongthong, T. (2006). The impact of total quality management on innovation: findings from a developing country. International Journal of Quality \& Reliability Management, 23(9), 1092-1117.

Tsai, W. (2001). Knowledge transfer in intraorganizational networks: Effects of network position and absorptive capacity on business unit innovation and performance. Academy of Management Journal, 44(5), 996-1004.

Yeh-Yun Lin, C. y Yi-Ching Chen, M. (2007). Does innovation lead to performance? An empirical study of SMEs in Taiwan. Management Research News, 30(2), 115-132.

(C) 2017 por los autores; licencia otorgada a la revista Escritos Contables y de Administración. Este artículo es de acceso abierto y distribuido bajo los términos y condiciones de una licencia Atribución-No Comercial 4.0 Internacional (CC BY-NC 4.0) de Creative Commons. Para ver una copia de esta licencia, visite https://creativecommons.org/licenses/by-nc/4.0/ 